\title{
FLEXIBLE, SKIN COUPLED MICROPHONE ARRAY \\ FOR POINT OF CARE VASCULAR ACCESS \\ MONITORING
}

\author{
By \\ BINIT PANDA
}

Submitted in partial fulfilment of requirements

for the degree of Master of Science

Thesis Advisor: Dr. Soumyajit Mandal

Department of Biomedical Engineering

CASE WESTERN RESERVE UNIVERSITY

August, 2019 


\title{
CASE WESTERN RESERVE UNIVERSITY SCHOOL OF GRADUATE STUDIES
}

\author{
We hereby approve the thesis of

\section{Binit Panda} \\ for the degree of Master of Science* \\ Committee Chair \\ Soumyajit Mandal \\ Committee Member \\ Dominique Durand \\ Committee Member \\ Colin Drummond \\ Committee Member \\ Steve Majerus
}

Date of Defense

May 16, 2018

*We also certify that written approval has been obtained for any proprietary material contained therein. 
Dedicated to the memory of my mother, Jyotirmayee 


\section{Acknowledgments}

I would like to offer my special thanks to Dr. Soumyajit Mandal and Dr. Steve Majerus for giving me this opportunity to work on this project and for the continual guidance and inspiration. I would furthermore like to thank Dr. Dominique Durand and Colin Drummond for their continuing help and support.

I would like to thank Brian, Brett, Ian and Steve for sharing the lab space with me and for the conversations we had during my period in the lab. Basement research lab would be different without your company.

I would not be able to achieve this without the support of my father, whether being financial or emotional. Thank you for always encouraging me and supporting me when I needed the most (semester 1). I want to thank my brother and uncle for the love and endless calls which made this journey of studying abroad bearable.

Finally, I want to thank Steve again for not only being an advisor but also a mentor and finding time to train me and leading my path through difficult times. Your confidence and motivation kept me going.

In closing, I would like to particularly acknowledge that this work was funded by the U.S. Department of Veterans Affairs Rehabilitation Research and Development Service, the Advanced Platform Technology Center of the Louis Stokes Cleveland Veterans Affairs Medical Center, and Case Western Reserve University under grant RX001968-01. 


\section{Table of Contents}

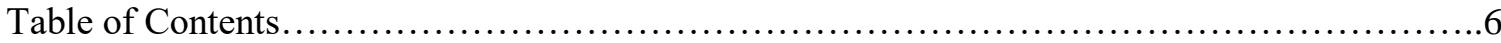

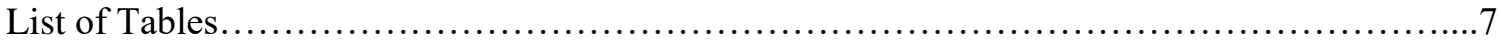

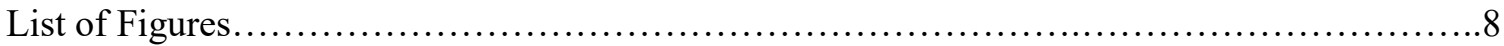



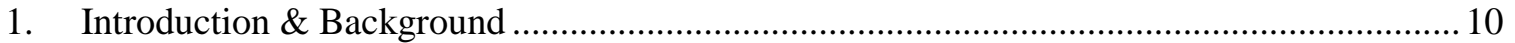

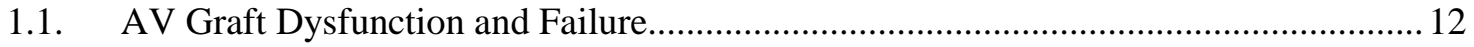

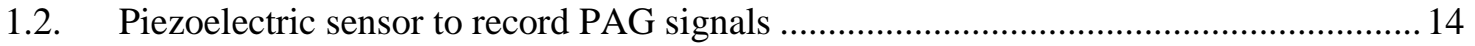

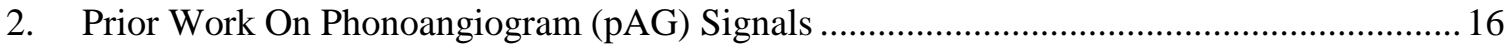

2.1. Effect of Stenosis and Blood Flow on Bruit Spectra and Intensity ............................... 16

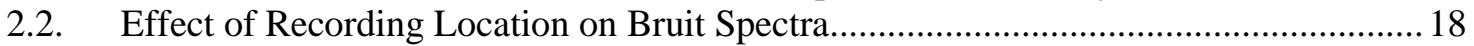

2.3. Stenosis Severity Classification from PAG Signal Analysis ........................................ 18

2.4. Comparison of Phonoangiogram signals from previous publications ......................... 20

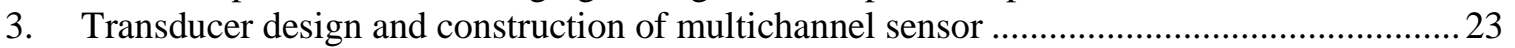

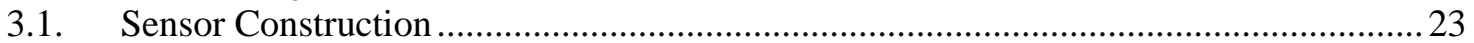

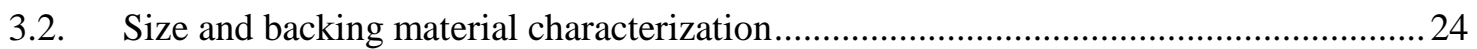

3.2.1. Frequency Response and Signal to Noise Ratio Calculation ....................................25

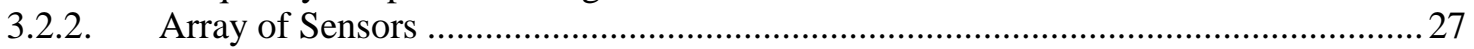

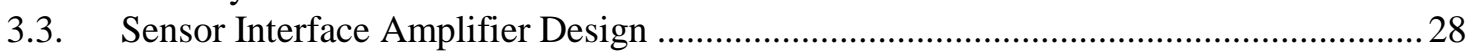

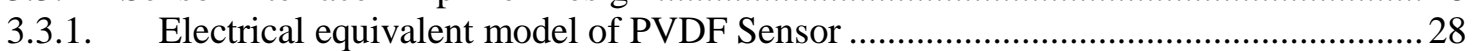

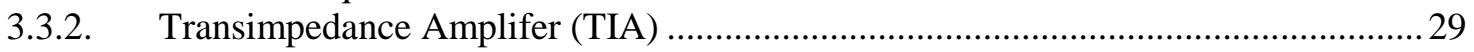

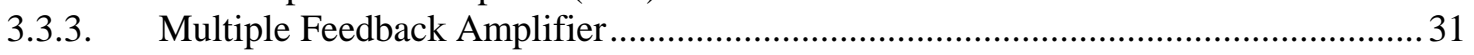

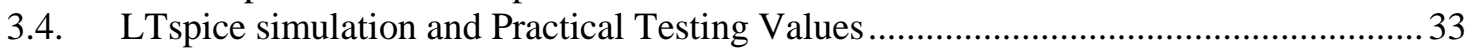

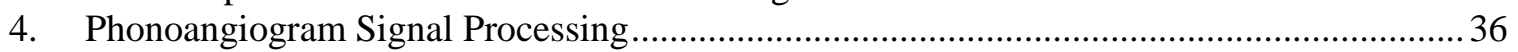

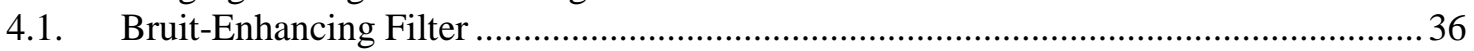

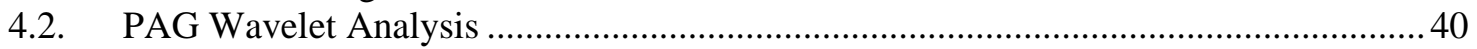

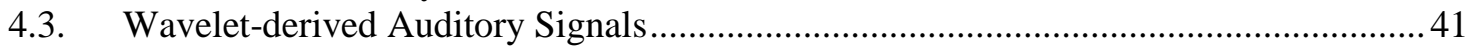

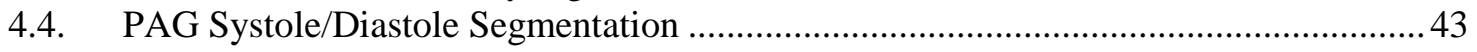

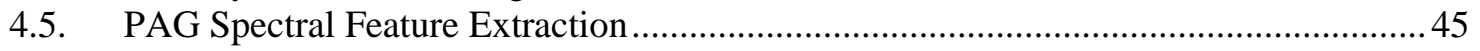

5. Detection of Vascular access stenosis location and severity in vitro .................................... 47

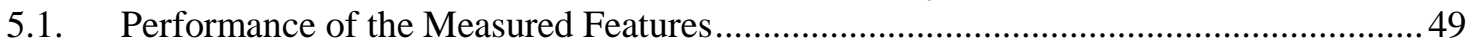

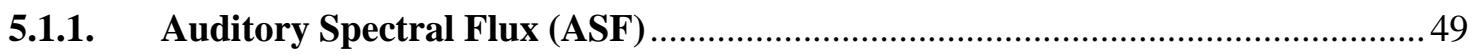

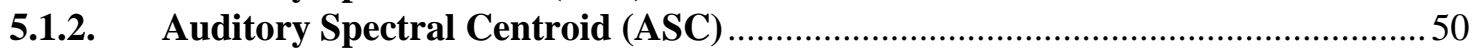

5.2. Threshold-based phonoangiographic detection of vascular access stenosis ..................53

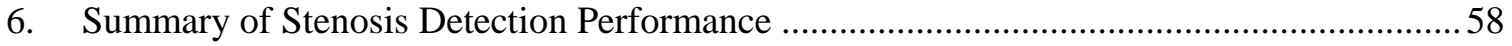

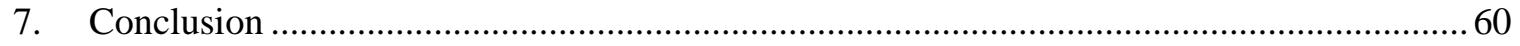

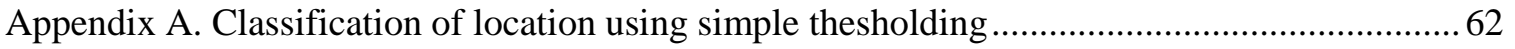

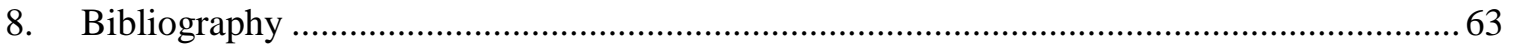




\section{List of Tables}

TABLE 1: FREQUENCY RANGES AFFECTED BY STENOSIS ………..........................................17

TABLE 2: PREVIOUSLY- PUBLISHED PAG STENOSIS CLASSIFIER METHODS ............................19

TABLE 3: MEASURED SNR OF PVDF SENSORS, MICROPHONE AND STETHOSCOPE ................26

TABLE 4: MODEL PARAMETER VALUES OF THE PVDF SENSOR ……….....................................29

TABLE 5: VALUES OF MULTIPLE FEEDBACK AMPLIFIER COMPONENTS ...................................32

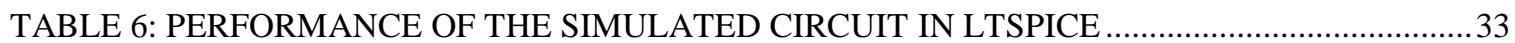

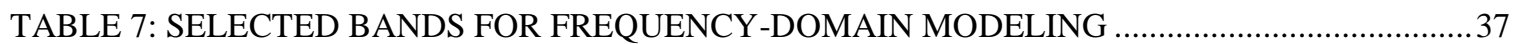



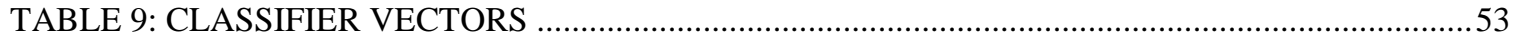

Table 10: PERFORMANCE OF SYSTOLE ASC MEAN FEATURE ..................................................55

Table 11: PERFORMANCE OF ASC DIFFERENCE BETWEEN SYSTOLE AND DIASTOLE ..............55

Table 12: PERFORMANCE OF SYSTOLE ASF RMS …………..........................................................56

Table 13: PERFORMANCE OF SYSTOLE ASC MEAN MULTIPLIED WITH SYSTOLE ASF RMS ...57 


\section{List of Figures}

Figure 1: Hemodialysis treatment uses an extracorporeal blood circuit to filter blood, removing impurities and excess fluid. [National Institute of Diabetes and Digestive and Kidney Disease, online at https://www.niddk.nih.gov/health-information/kidneydisease/kidney-failure/hemodialysis]

Figure 2: Hemodialysis a) vascular access fistula and, b) vascular access graft during dialysis treatment. [Image by the Mayo Foundation for Medical Education and Research. All rights reserved]

Figure 3: A typical central venous catheter (CVC) used for dialysis. [Image available online at [https://www.apexkidneyfoundation.org/patienthelp/patient-help-vascular-access.php] - 11

Figure 4: PAG waveform from a vascular access with a high degree of stenosis [2]. ------------ 21

Figure 5: PAG signals in the time domain for a patient with a vascular graft [44]. ---------------- 21

Figure 6: Phonoangiogram recorded using a custom piezoelectric sensor (discussed later) from a vascular access phantom. 22

Figure 7: Aggregate power spectrum of phantom PAGs. The spectrum is approximately flat in the range of $1000-2500 \mathrm{~Hz}$. 22

Figure 8: Cross-sectional view of the sensor showing the PVDF film sandwiched between two electrodes and a layer of PDMS on the skin-facing side. (B) Layout of a sensor test board containing $2,4,8$, and 16- $\mathrm{mm}$ diaphragm sizes.

Figure 9: Typical frequency response curve for the microphone, which has a flat response until 2 kHz. ----- 25

Figure 10. Test setup used for frequency response measurements. -

Figure 11. Sensors with silicone gel backing had the highest SNR and performed well at all the three test frequencies.

Figure 12: Prototype PAG sensor array based on 2-mm diameter PVDF microphones on polyimide substrate with silicone skin-coupling interface (A). PAG array is thinner than $1 \mathrm{~mm}(B)$ and flexible to conform to vascular access shape $(C)$. ------------ 28

Figure 13: Electrical equivalent model of the PVDF sensor. ---

Figure 14: Circuit diagram of a transimpedance amplifier (TIA). ------------------------ 29

Figure 15: Circuit diagram of a multiple feedback amplifier. -------------------------------------- 31

Figure 16: LTspice simulated circuit diagram. --------------------------------------------- 33

Figure 17: Simulated small-signal frequency response of the TIA. ----- 34

Figure 18: Simulated small-signal frequency response of the multiple feedback amplifier. ------ 34

Figure 19: Measured small-signal frequency response of the TIA.------ 35

Figure 20. Measured small-signal circuit frequency of the multiple feedback amplifier. ---------- 35

Figure 21: PAGs are processed in frames using a bruit-enhancing filter to produce an enhanced PAG $\mathrm{X}_{\mathrm{BEF}}[\mathrm{n}]$ and the bruit power envelope $\mathrm{E}_{\mathrm{BEF}}[\mathrm{n}]$. The BEF breaks the discrete cosine transform of $\mathrm{X}[\mathrm{n}]$ into $\mathrm{N}$ sub- bands. Sub-bands are modeled using linear predictive coding and recombined with weights $\mathrm{W}_{\mathrm{N}}$ to produce a bruit envelope $\mathrm{E}_{\mathrm{BEF}}[\mathrm{n}]$.

Figure 22: PAG spectra from chronic dialysis recipients (1,126 recordings). Spectra were consistent across patients over $2-12$ months of recordings. The average spectrum is (black line) revealed 4 bands with unique spectral features [54].

Figure 23: Spectrogram of a PAG without the bruit-enhancing Filter (A), and with the filter (B).

Figure 24: Spectrogram of artificially generated test waveform (top) with 6 single-tone frequencies from 100-1500 Hz. The ASF curve (bottom) shows a spike at every change of frequency, approximating the spectral first derivative.---------------- 42 
Figure 25: Spectrogram of artificially generated test waveform (top) with 6 single-tone frequencies from 100-1500 Hz. The ASC curve (bottom) describes the frequency of the sine wave at each time point.

Figure 26: Segmentation is performed on ASF with a 2-stage approach. Stage 1 uses threshold crossing to locate initial start and end of a segment. Stage 2 filters out spurious segments caused by recording noise peaks.

Figure 27. When degree of stenosis (DOS) is 30\% (A), the power is confined to the systole phase (white box). For high DOS of $80 \%$ (B), turbulent flow increases the power in systole and diastole (black box) segments.

Figure 28. 10-85\% stenosis AVG phantoms used to reproduce bruits were constructed using banded 6-mm silicone tubing (A) embedded in tissue-mimicking silicone rubber to a depth of $6 \mathrm{~mm}(\mathrm{~B})$. Contrast angiography validated recording site locations (C), with computed CT slices used to calculate the degree of stenosis (D).

Figure 29: The flexible PVDF sensor array was tested on 10-85\% stenosis phantoms with variable flow. PAG systole spectral flux RMS (regardless of DOS or flow) was different at each recording site. A sharp drop in $\mathrm{ASF}_{\mathrm{RMS}, \mathrm{S}}$ at Location 2 suggests the presence of stenosis within a distance of $1 \mathrm{~cm}$ proximal to the direction of the flow.

Figure 30: As the Degree of stenosis increased, the $\mathrm{ASF}_{\mathrm{RMS}, \mathrm{S}}$ value also increased. This trend can be seen while moving from mild to moderate to severe DOS: there is a monotonic increase in the $\mathrm{ASF}_{\mathrm{RMS}, \mathrm{S}}$ irrespective of the location.

Figure 31: The flexible PVDF sensor array was tested on 10-85\% stenosis phantoms with variable flow. PAG spectra (regardless of DOS or flow) (A) had distinct auditory spectral centroid (ASC) values at $1 \mathrm{~cm}$ spacings both proximal and distal to stenosis. ------ 51

Figure 32: Low DOS (A) showed a greater peak-valley change in auditory spectral centroid (ASC) compared to high DOS (B). The difference in systole and diastole is computed after ASF-based segmentation.

Figure 33. The difference between Systole ASC mean and Diastole ASC mean is inversely related to the DOS. As the DOS increases, the flow becomes turbulent and the difference between Systole and Diastole ASC mean decreases.

Figure 34: ROC curve of Systole ASC mean feature. Greatest area-under-the curve is achieved at Location 3 just distal to the stenosis.

Figure 35: ROC curve of ASC difference between systole and diastole. Greatest area-under-the curve is achieved at Location 3 just distal to the stenosis.

Figure 36: ROC curve of systole ASF RMS. Greatest area-under-the curve is achieved at Location 4 distal to the stenosis. 56

Figure 37: ROC curve of Systole ASC mean multiplied with systole ASF RMS. Greatest areaunder-the curve is achieved at Location 4 distal to the stenosis.

Figure 38: Difference in systole ASC mean from adjacent locations. 


\section{Flexible, Skin Coupled Microphone Array \\ for Point of Care Vascular Access \\ Monitoring}

Abstract

By

\section{BINIT PANDA}

The main goal of this thesis is to develop a flexible and body-conformal sensor for vascular access stenosis characterization. Vascular accesses are monitored through physical examination by medical personnel. These examinations are time consuming and subjective, thus creating a challenge for long term monitoring and an opportunity for automated analysis. In our method, we record phonoangiograms from vascular access phantoms through a custom-made flexible array of piezoelectric sensors. These signals are processed through an autoregressive bruit- enhancing filter which used a nonlinear, sub-band frequency-domain linear prediction approach to amplify the systolic pulses of the blood flow and associated stenosis related turbulence while attenuating interpulse intervals and artifacts due to ambient noise. Features like auditory spectral centroid (ASC) and auditory spectral flux (ASF) are extracted from the wavelet coefficients which are computed over 6 octaves with 12 voices/octave, starting at scale 3. Finally, we show that this piezoelectric sensor in combination with signal processing techniques can i) localize stenosis within $1 \mathrm{~cm}$ of the actual location, and ii) differentiate the degree of stenosis into three grades (mild, moderate and severe) in vitro. These results were confirmed with statistical analysis and the performance of the extracted features was also tested with a threshold-based stenosis detection classifier to mimic a clinical screening scenario aimed at identifying at-risk patients. 


\section{INTRODUCTION \& BACKGROUND}

Hemodialysis is a treatment to filter waste and excess water from the blood for people who have acute or chronic kidney failure. During the treatment, arterial blood is pumped into a dialyzer and the filtered blood is returned to the venous system as shown in Figure 1. To improve hemodialysis, permanent vascular access is often obtained using arteriovenous (AV) fistulas, AV grafts or central venous catheters (Figure 2 and 3) [1], [2]. Long-term dialysis success and cost is dependent on maintaining the patient's vascular access - the so called "Achilles Heel" of hemodialysis [3]. Access dysfunction accounts for 20-30\% of hospital visits for dialysis patients and the loss of access patency can double their mortality risk. The predominant causes of access dysfunction are stenosis (vascular narrowing) and thrombosis (vascular occlusion), which have a combined incidence of $66-73 \%$ in arteriovenous fistulas (AVFs) and $85 \%$ in arteriovenous grafts (AVGs). Further, 23-55\% of AVFs never mature (supporting sufficient blood flow) due to venous inflow stenosis [3]. Maintenance of vascular access is therefore a key objective of the Kidney Disease Outcomes Quality Initiative (KDOQI) and dialysis care in general [1][4].

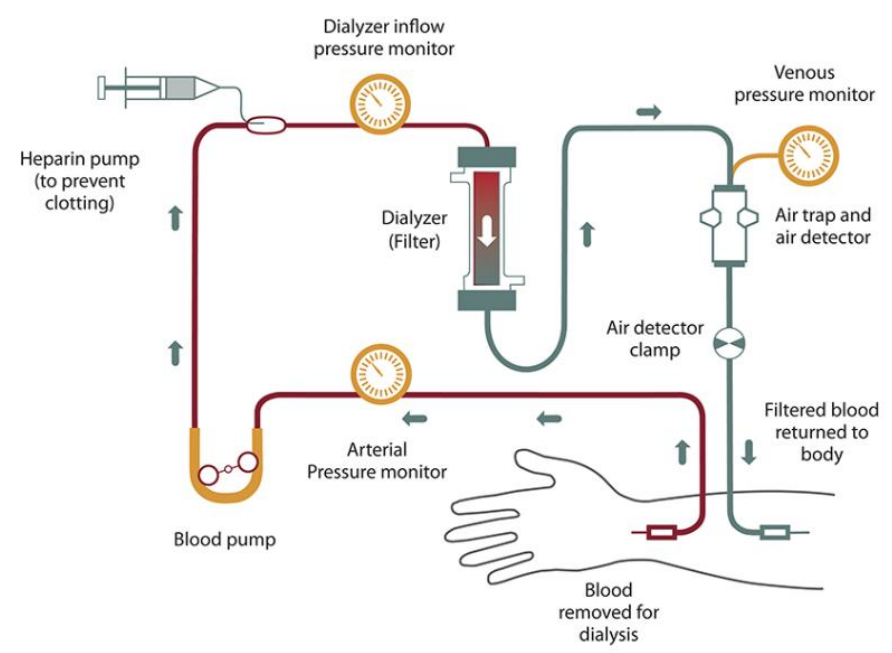

Figure 1: Hemodialysis treatment uses an extracorporeal blood circuit to filter blood, removing impurities and excess fluid. [National Institute of Diabetes and Digestive and Kidney Disease, online at https://www.niddk.nih.gov/health-information/kidney-disease/kidneyfailure/hemodialysis] 

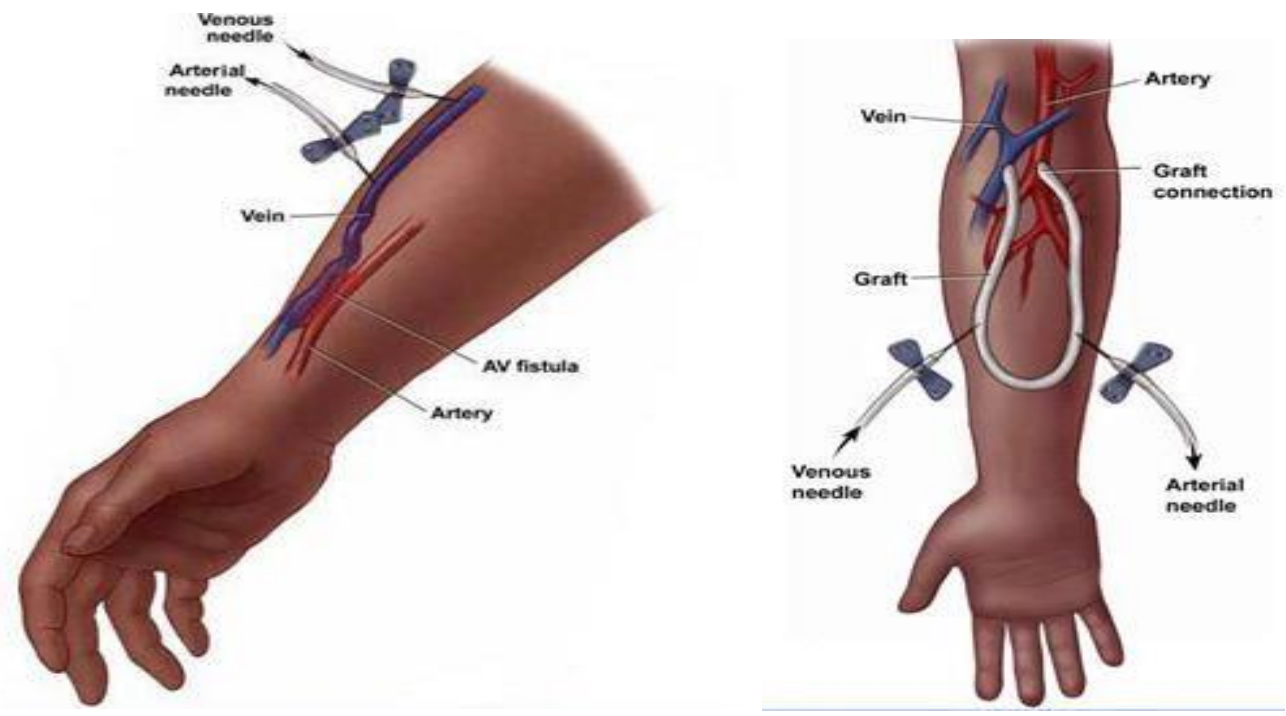

Figure 2: Hemodialysis a) vascular access fistula and, b) vascular access graft during dialysis treatment. [Image by the Mayo Foundation for Medical Education and Research. All rights reserved]

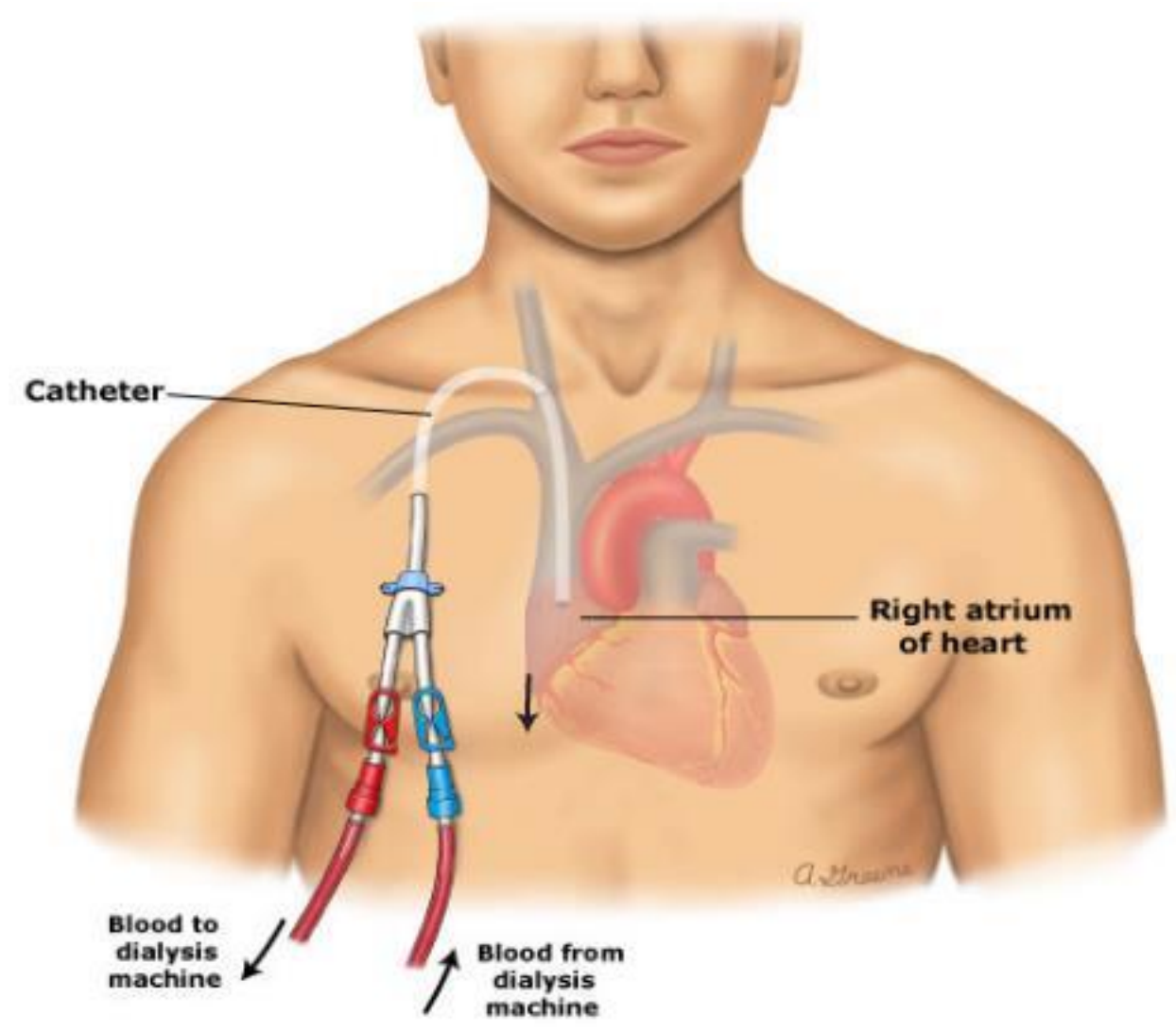

Figure 3: A typical central venous catheter (CVC) used for dialysis. [Image available online at [https://www.apexkidneyfoundation.org/patienthelp/patient-help-vascular-access.php] 


\subsection{AV Graft Dysfunction and Failure}

Access failure via thrombosis secondary to stenotic lesions in the first year of use is very high [5]. Among 649 patients with new AVGs enrolled in the Dialysis Access Consortium study, $77 \%$ developed stenosis or thrombosis within the first year [5]. Technical issues were the main cause of immediate failures of AVGs which were often related to inadequate anastomosis creation due to severe arterial calcification [6]. A large, single-center study observed immediate failure in $5.8 \%$ of upper extremity AV grafts and $12.7 \%$ of thigh grafts [7]. Postoperative graft failures are typically related to stenotic vascular lesions, most commonly affecting the venous outflow anastomosis due to neointimal hyperplasia[6]. In a large single-center observational study of 310 AV grafts, $80 \%$ of graft failures were due to thrombosis, $14 \%$ were due to infection, and 3\% were due to a large pseudo aneurysm with impending rupture. The remainder were caused by intractable central vein stenosis requiring AV graft ligation, and distal ischemia [8][9].

Monitoring of hemodialysis AVFs and AVGs for stenosis is performed using clinical exam and non-invasive surveillance methods. Non-invasive monitoring methods have a relatively high positive predictive value (PPV) for detecting more stenosis above 50\% [10]. A randomized, controlled trial found that abnormalities detected by clinical monitoring had a $70 \%$ PPV for hemodynamically significant AVG stenosis compared with Duplex ultrasound, which had a $80 \%$ PPV [10].

Clinical monitoring consists of assessments that are readily available from physical examination of the access and laboratory studies in the dialysis unit [11]. These include:

1. Physical examination and auscultation of the graft extremity, which includes absence of thrill, abnormal auscultation, or edema distal to the graft. 
2. Difficulties experienced during the dialysis session like prolonged bleeding from the needle site or difficulty in cannulation.

3. An unexplained decrease in dialysis treatment adequacy over time on a constant dialysis prescription.

The major advantage of clinical monitoring over graft surveillance is the low cost, because monitoring data is generated naturally through the administration of dialysis treatment. However, the sensitivity of clinical monitoring depends on the skill of the dialysis staff to detect access abnormalities in real time. This makes it very subjective and prone to misinterpretation of the results.

An alternative to clinical monitoring is a coordinated program of vascular access surveillance. Surveillance requires specialized equipment or trained staff and is more consistent and reproducible. To overcome the limitation of clinical monitoring, several quantitative surveillance methods have been developed, but are not always reimbursed in the United States. Generally, surveillance adds more cost to dialysis treatment, although some studies indicate net cost savings when accounting for reduced risk of patient hospitalization arising from undetected vascular access dysfunction.

Three prevalent modes of access surveillance include [11]:

1. Dialysis venous pressures

2. Flow monitoring

3. Duplex ultrasound

In static dialysis venous pressure, a manometer is hooked to the arterial dialysis needle before starting the dialysis pump. The intra-graft pressure is normalized to the systemic blood pressure, and expressed as static venous pressure ratio (SVPR). One would expect a progressive increase in SPVR as the magnitude of stenosis increases [12]. 
Flow monitoring works on the principle of saline- or thermo-dilution to estimate blood flow through access. In thermodilution, cold saline is injected rapidly through the arterial port while the venous and arterial lines are reversed in orientation. By measuring the area under the temperature curve, the computer can calculate the access blood flow [13]. Saline dilution is used in a similar manner, in which a bolus of saline is injected and ultrasonic detection of saline removal is measured such that area-under-the-curve measurement is performed. The net result of dilution techniques is to characterize the true access flow rate. An access blood flow $<600$ $\mathrm{ml} / \mathrm{min}$ or a decrease in flow by $>25 \%$ from baseline is predictive of significant access stenosis [14].

Duplex ultrasound monitoring of vascular access primarily measures peak systolic velocity (PSV) at anastomoses and sites with visual stenosis. It can also measure the blood flow as a secondary supportive indicator of graft stenosis [15], [16]. However, unlike static dialysis pressures and other monitoring methods, this test is often performed in a radiologic outpatient setting separate from the dialysis treatment center. Further, the sensitivity of this technique is partially dependent on the expertise of the clinician administering the exam.

Existing methods of vascular access surveillance have high chances of predicting stenosis growth. However, they are invasive and highly dependent on skills. These surveillance methods are very late in detecting early onset of any problem. Thus they are ineffective in changing the outcome. This gives an opportunity to develop a sensor that can be used for screening potential high-risk patients. Treating these patients earlier can result in better treatment outcomes.

\subsection{Piezoelectric sensor to record PAG signals}

When blood flows through a constricted vessel, the resulting jet flow induces a turbulent flow and a pressure fluctuation in the blood vessel wall [17]. This produces distinct sounds known as arterial bruits which can be heard with a stethoscope. Auscultation for bruits is a 
mainstay of vascular access physical exams, but is increasingly skipped due to time constraints and the relative skill required to interpret vascular bruits. Recording and mathematical analysis of bruit sounds (a technique called phonoangiography) can be used as a new tool for rapid and objective access monitoring [17]-[21]. Phonoangiograms (PAGs) contain a unique acoustic spectrum that correlates strongly with degree of stenosis (DOS), and data interpretation can be automated [22].

A piezoelectric sensor uses the piezoelectric effect whereby mechanical deformation of the material produces a proportional electrical charge. Polyvinylidene fluoride (PVDF) is a piezoelectric polymer that is mechanically flexible and sensitive to applied strain, while remaining thin, light-weight and inexpensive. These properties make it suitable for portable and disposable sensing devices. PVDF has been widely used for different biomedical signal detection applications. For example, Kalange et al. [23] used it for detection of human pulse. Similarly Teague et al. [24] compared the performance of piezoelectric film to electret microphones and MEMS in obtaining joint sounds in healthy collegiate athletes during unloaded flexion/extension. Hallikar et al. compared the performance of PVDF and lead zirconate titanate (PZT 5A) in designing an Artificial Basilar Membrane to mimic the properties of the cochlea [25]. Their simulation results confirmed the suitability of PVDF material for frequency separation in the range of $1 \mathrm{kHz}$ to $10 \mathrm{kHz}$. In [26], Lei et al. used Polydimethylsiloxane (PDMS) to coat the PVDF film to help damp vibrations and reduced the signal noise generated in dynamic conditions. Chiu et al. in [27] also used this technique to develop a sensor patch for simultaneous heartbeat and respiration monitoring. In [28], Jiang et al. discussed a technique to make the PVDF film insensitive to bending by applying PDMS to both sides of the PVDF film. This helped in detecting heartbeat more accurately while a PVDF film with PDMS on one side made it more sensitive to bending and it helped in detecting respiration. 


\section{PRIOR WORK ON PHONOANGIOGRAM (PAG) SIGNALS}

Although PAGs have been studied over several decades, there is a relative sparsity of work and widely-varying descriptions of relevant spectral properties in functional and dysfunctional vascular accesses. The underlying theme of prior work has been to identify how PAG spectra change relative to the degree of stenosis (DOS) and the recording location relative to the stenosis. DOS is defined as the ratio of the stenosed cross-sectional area of the blood vessel to the proximal (non-stenosed) lumen diameter. The clinical standard to locate stenosis and calculate DOS is angiography, in which X-ray scans are used with radiocontrast in the blood for precise imaging of the vascular geometry [29]. DOS is typically estimated within $10 \%$ due to the precision attainable in clinical environments.

Previous studies have analyzed PAGs from humans, from vascular bench phantoms, and from computer simulations of blood flow. Here, we describe three topics which have been studied previously: the spectral properties of PAGs in normal and stenosed cases, the impact of recording location on PAG spectra, and classification of DOS severity based on PAG analysis.

\subsection{Effect of Stenosis and Blood Flow on Bruit Spectra and Intensity}

Much early work in PAG analysis used Fourier transform and other time-invariant analyses

to quantify the frequency content of PAGs. These methods represented the combined frequencies generated during systolic and diastolic phases of turbulent blood flow. Because clinical interpretation of pathologic bruits relies on detecting a high-pitched whistling character, it was hypothesized that stenosis would shift spectral power within a certain frequency band [2]. Although all studies agree that the frequency range of interest is in the $0-1000 \mathrm{~Hz}$ band, identification of specific frequency bands varied widely. More recent work has abandoned simple spectral analysis in favor of more complex multi-feature classifiers (described below) [2], [22], [30]-[34]. 
TABLE 1: FREQUENCY RANGES AFFECTED BY STENOSIS

\begin{tabular}{|l|l|}
\hline Reference & Frequency ranges affected by stenosis \\
\hline Chen 2013 [35] & Specific bands of 130-260, 250-500, and 550-800 Hz \\
\hline Akay 1993 [36] & $300-800 \mathrm{~Hz}$ \\
\hline Obando, et al. 2012 [37] & $200-600 \mathrm{~Hz}$ \\
\hline Vasquez, et al. 2009 [38] & $300-500,200-800,250-1000 \mathrm{~Hz}$ \\
\hline Mansy, et al. 2005 [33] & $300-600 \mathrm{~Hz}$ \\
\hline Wang 2011 [39] & $700-800 \mathrm{~Hz}$ after signal processing \\
\hline Sato, et al. 2006 [40] & $20-800 \mathrm{~Hz}$ \\
\hline Wang, et al. 2014 [34] & $200-600 \mathrm{~Hz}$ \\
\hline Chen, et al. 2014 [41] & $40-800 \mathrm{~Hz}$ \\
\hline Gram, et al. 2010 [42] & $625-750 \mathrm{~Hz}$ and $875-1000 \mathrm{~Hz}$ \\
\hline Sung, et al. 2014 [2] & $50-800 \mathrm{~Hz}$ \\
\hline Todo, et al. 2012 [43] & $51-270 \mathrm{~Hz}$ \\
\hline Chen 2013 [44] & $25-800 \mathrm{~Hz}$ \\
\hline Mungia, et al. 2009 [45] & $200-1000 \mathrm{~Hz}$ \\
\hline Grochowina, et al. 2014 [46] & 150 and 350-400 Hz \\
\hline Rousselot 2014 [47] & $70-800 \mathrm{~Hz} ;$ specific bands at 180-300,310-390, 440-700 Hz \\
\hline
\end{tabular}

Moreover, although most studies agreed that DOS caused enhanced high-frequency spectral power [2], [34]-[37], [39], [40], [42]-[44], [46], [47], one prominent study described an opposite effect [33]. This was perhaps due to the effect of DOS to reduce blood flow because blood pressure is regulated; reduced flow would produce lower overall bruit amplitudes due to reduced turbulence for low blood flow [48], [49]. Studies have also demonstrated that stenosis can increase the intensity (amplitude) of PAGs [43], [44], [27]. Amplitude-based analysis, however, is affected by the mechanical-acoustical coupling of the skin and recording transducer, and can be affected by skin scratch artifacts. Therefore, most work has focused on spectral analysis.

Despite the disagreement in the precise effect of stenosis on bruit spectra, however, these prior studies confirmed that stenosis definitively changes PAG amplitude and pitch. The change, however, could be an enhancement or a suppression of certain frequencies depending on the impact of stenosis on blood flow. Other patient-dependent variables such as PAG amplitude and the recording location relative to stenosis must also be accounted for, and are described below. 


\subsection{Effect of Recording Location on Bruit Spectra}

Compared to frequency analysis reports, there is significantly less disagreement on the effect of recording location on PAG spectra. This is partly due to the obvious property that a bruit cannot be detected when very far away from the location of stenosis (for example on the contralateral limb). Therefore, bruits can only be detected against the background physiologic sounds when close to the source of turbulent blood flow. Fluid dynamic simulations have established to a high degree of precision that stenosis induces turbulent flow at physiologic blood pressures, flow rates, and nominal lumen diameters of vascular accesses [48], [49]. These simulations have been confirmed by Doppler ultrasound measurements, which agree that turbulent flow is present at a distance of 2 - 5 times the diameter of the unoccluded tube distal of stenosis. The important distinction is that turbulence and increased pressure always occurs on the downstream, or venous, side of the stenosis. Therefore, bruits recorded proximally and distally to stenosis will have different frequency spectra due to stenosis turbulence. This effect was confirmed using dual-channel stethoscopes [30] . However, the acoustical influence of biomechanical properties and thickness of tissue over the vascular access varies between patients. Because tissue acts as a low-pass filter at auditory frequencies, it is presumed that the most accurate bruit recordings would be obtained in the $1-3 \mathrm{~cm}$ region distal to stenosis (assuming unoccluded tube diameter to be $6 \mathrm{~mm}$ ), where turbulent flow is maximal [48], [49].

\subsection{Stenosis Severity Classification from PAG Signal Analysis}

Many recent works have focused on the use of various classification methods based on spectro-temporal features. These techniques are promising because they more closely approximate how trained clinicians interpret bruits - through a combination of time-based and spectral-based observations. However, because of the complexities and wide dynamic range of PAGs, no single technique has been identified as superior. Some of these approaches leverage 
the pulsatile nature of blood flow, where a distinct change in spectra occurs in systole and diastole. Other approaches rely on clustering or machine learning strategies to combine several time-domain and spectral features. Because various techniques have been used, here we only summarize prominent examples [35], [44], [41], [42], [2], [34], [45], [39], [38].

TABLE 2: PREVIOUSLY- PUBLISHED PAG STENOSIS CLASSIFIER METHODS

\begin{tabular}{|c|c|c|}
\hline Reference & Classifier method & $\begin{array}{l}\text { Classifier details and } \\
\text { performance }\end{array}$ \\
\hline Chen 2013 [35] & $\begin{array}{l}\text { Burg autoregressive (AR) model } \\
\text { of frequency spectrum }\end{array}$ & $\begin{array}{l}\text { Stenosis causes increase of } \\
\text { frequency of fitted poles in } \\
\text { model }\end{array}$ \\
\hline Chen 2013 [44] & $\begin{array}{l}\text { Burg autoregressive model of } \\
\text { frequency spectrum and } \\
\text { fractional-order chaotic system }\end{array}$ & $\begin{array}{l}\text { Correlation }(0.38) \text { between } \\
\text { chaotic model output } \\
\text { parameter and degree of } \\
\text { stenosis reduction from } \\
\text { PAGs at venous site of } \\
\text { AVF }\end{array}$ \\
\hline Chen, et al. 2014 [41] & $\begin{array}{l}\text { Burg autoregressive model of } \\
\text { frequency spectrum and a fuzzy } \\
\text { petri net }\end{array}$ & $\begin{array}{l}\text { Stenosis can be graded by } \\
\text { Petri net into } 1 \text { of } 3 \text { classes }\end{array}$ \\
\hline Gram, et al. 2010 [42] & $\begin{array}{l}\text { Linear discriminant analysis } \\
\text { (LDA) }\end{array}$ & $\begin{array}{l}\text { Power distribution in } \\
\text { highest scale of discrete } \\
\text { wavelet transform relative } \\
\text { to power in other scales } \\
\text { allows detection of stenosis }\end{array}$ \\
\hline Sung, et al. 2014 [2] & $\begin{array}{l}\text { Principal component analysis } \\
\text { (PCA) based on two-dimensional } \\
\text { Gaussian distribution }\end{array}$ & $\begin{array}{l}\text { Covariance between } \\
\text { spectral centroid and } \\
\text { spectral flux correlate most } \\
\text { strongly with stenosis }\end{array}$ \\
\hline Wang, et al. 2014 [34] & $\begin{array}{l}\text { Radial basis function (RBF) } \\
\text { neural network }\end{array}$ & $\begin{array}{l}\text { S-transform used for } \\
\text { feature detection; time } \\
\text { characteristics of spectra } \\
\text { during systolic pulse are } \\
\text { essential to analyze } \\
\text { accurately }\end{array}$ \\
\hline Mungia, et al. 2011 [45] & $\begin{array}{l}\text { Principal component analysis and } \\
\text { sequential forward selection } \\
\text { algorithm }\end{array}$ & $\begin{array}{l}\text { Best feature was energy } \\
\text { difference between wavelet } \\
\text { scales at } 500-1000 \mathrm{~Hz} \text { and } \\
250-500 \mathrm{~Hz}\end{array}$ \\
\hline Wang, et al. 2011 [39] & Narrowband power threshold & $\begin{array}{l}\text { Stenosis detectable if power } \\
\text { in the } 700-800 \mathrm{~Hz} \text { band } \\
\text { exceeds } 1 / 15^{\text {th }} \text { of the } \\
\text { maximum power }\end{array}$ \\
\hline $\begin{array}{l}\text { Vasquez, et al. } 2009 \\
\text { [38] }\end{array}$ & $\begin{array}{l}\text { Support vector machine (SVM) to } \\
\text { classify between wavelet scale } \\
\text { energies }\end{array}$ & $\begin{array}{l}\text { Normalized scale energies } \\
\text { and logarithmic transform }\end{array}$ \\
\hline
\end{tabular}




\begin{tabular}{|l|l|l|}
\hline & & $\begin{array}{l}\text { allowed for use of linear } \\
\text { kernel }\end{array}$ \\
\hline
\end{tabular}

Several limitations of these early-stage studies should be noted. First, all these studies recruited pathologic patients, i.e. patients who were already suspected for vascular access dysfunction and who were scheduled for surgical interventions. Therefore, there is a strong bias in interpretation of results, and limited opportunity for confirmation of classifier algorithms on a randomly selected test set. Second, while classifiers were trained to determine DOS from PAG features, most studies have reported on the performance of a binary classifier, i.e. detecting if a vascular access has DOS $>50 \%$. This detection level is clinically important because it is at the level at which stenoses become hemodynamically significant and surgical intervention may be justified [2]. Therefore, although many methods sought to describe DOS directly from PAG features, the primary utility of classification would be to simply identify which patients have DOS $>50 \%$ to enable the use of clinical imaging for a more detailed investigation of pathology.

\subsection{Comparison of Phonoangiogram signals from previous publications}

This section describes PAG signal from earlier work. We will show the similarities between these signals and the one from our project. The goal is to introduce readers to a PAG signal and discuss about the method in later sections. 


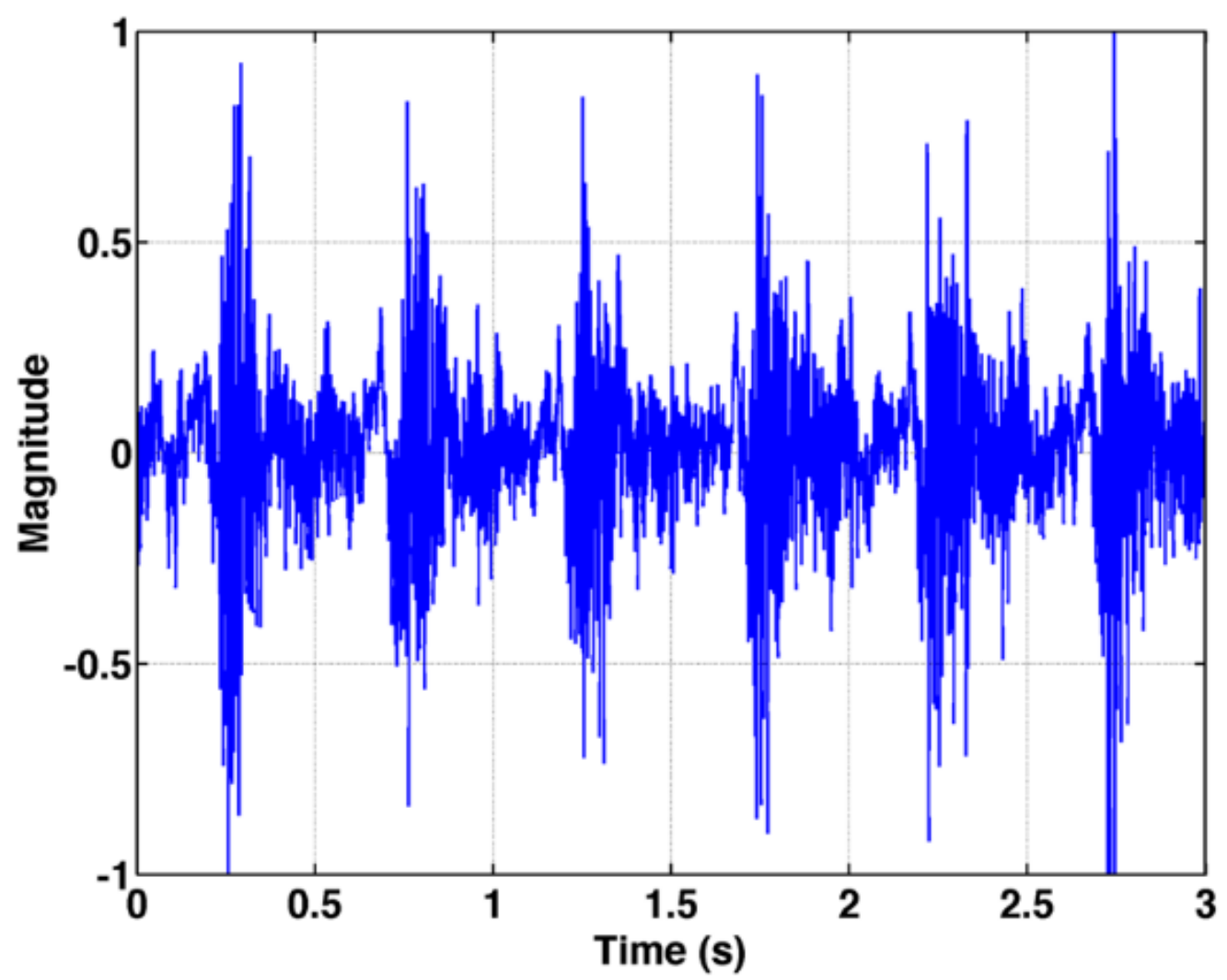

Figure 4: PAG waveform from a vascular access with a high degree of stenosis [2].

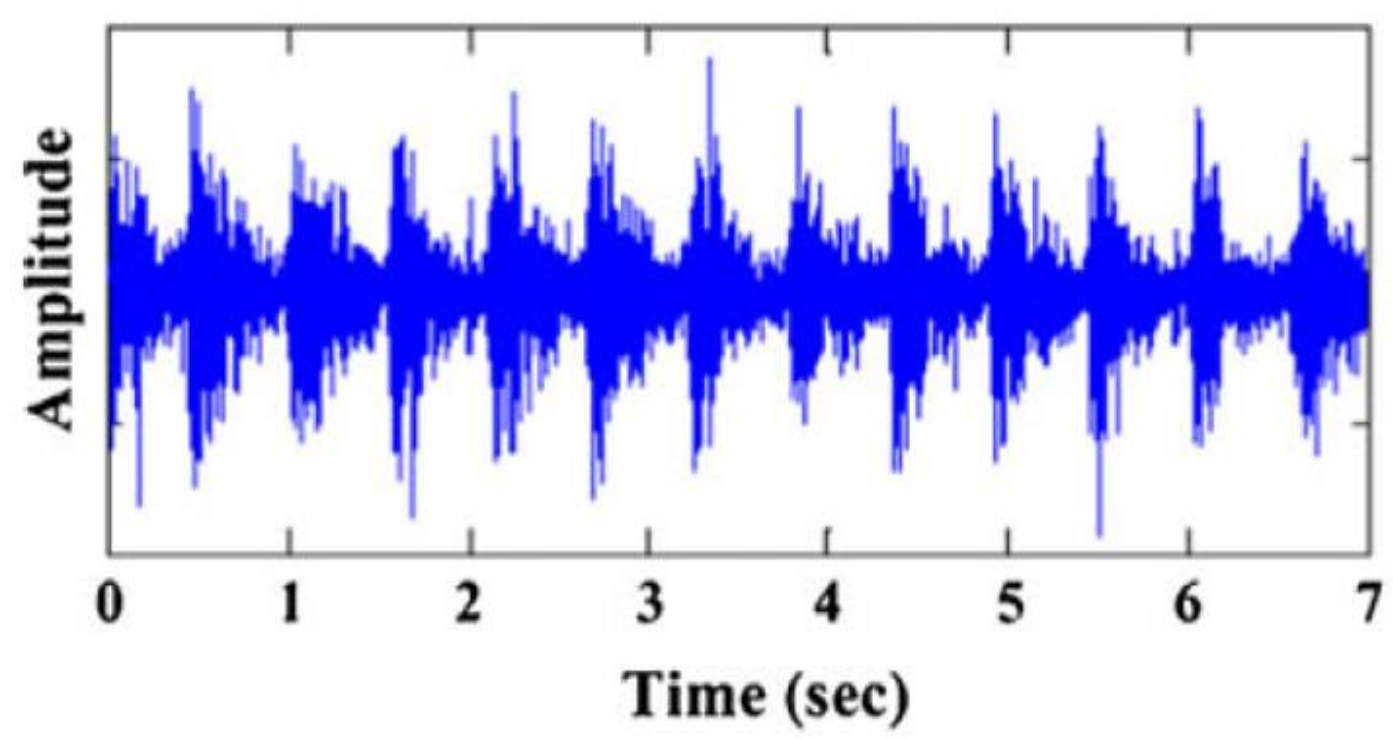

Figure 5: PAG signals in the time domain for a patient with a vascular graft [44]. 
Figure 4 shows a PAG signal from a patient recorded at A- site. The degree of stenosis is high in this condition while Figure 5 shows a PAG from a patient with the vascular draft. Both of these signals are recorded by different groups around the world. However, qualitatively we can say that these signals are similar to each other. Now, we will show a PAG signal recorded from the sensor which will be introduced in later sections (Figure 6). As the flow rate is slower in the phantom setup than the real patient, we only see 10 pulses in 10 seconds. The pulse structure looks exactly the same as from patients. Figure 7 shows the average power spectrum of phantoms studied in this project. We can see the flat response of the sensor in recording PAG signal. Now that we have introduced how a PAG looks like, we will talk more about the sensor and the experiments in next section.

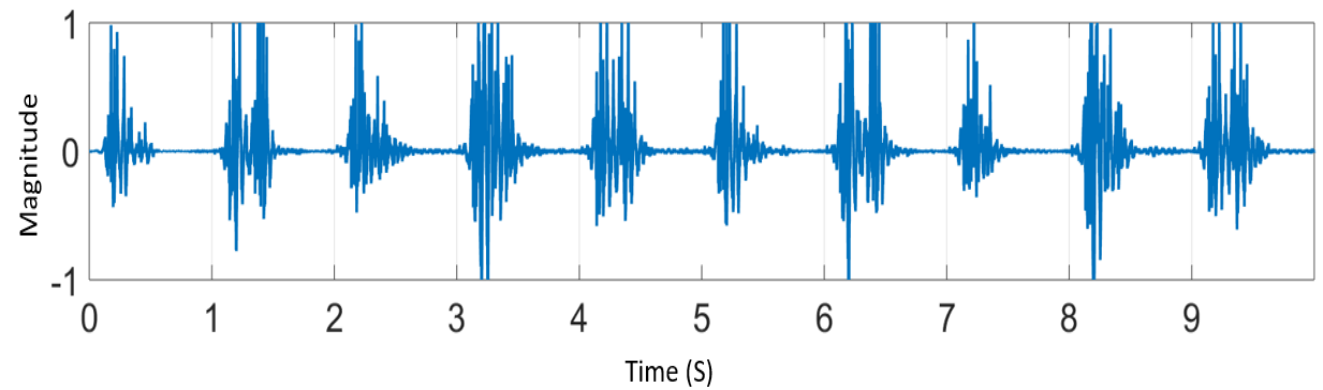

Figure 6: Phonoangiogram recorded using a custom piezoelectric sensor (discussed later) from a vascular access phantom.

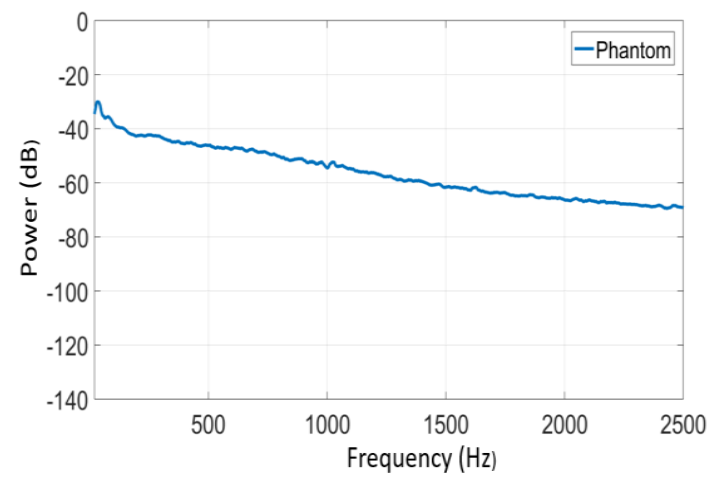

Figure 7: Aggregate power spectrum of phantom PAGs. The spectrum is approximately flat in the frequency range of $1000-2500 \mathrm{~Hz}$ 


\section{TRANSDUCER DESIGN AND CONSTRUCTION OF MULTICHANNEL SENSOR}

This chapter describes the sensor construction and analysis of frequency response using different sensor sizes and acoustic backing materials. We will also discuss the amplifier circuit used for amplification of the sensor signal.

\subsection{Sensor Construction}

Sensors were constructed using silver ink metallized PVDF film (Measurement Specialties MEAS) with a thickness of $28 \mu \mathrm{m}$. Films were cut into required sizes by a laser cutter (Versa LASER, Models VLS2.30 \& VLS 3.50) in two steps. First, the outer $0.5 \mathrm{~mm}$ of metallization was removed from one sensor surface by raster-scanning at $14 \%$ power and $30 \%$ speed to weaken the metal ink-PVDF bond. Weakened regions were exfoliated by tape prior to cutting through the PVDF film to prevent shorting of the metal across the cut film. A final thickness cut made at $17 \%$ power and $100 \%$ speed released each transducer element.

Transducers were sandwiched between two identical printed circuit boards (PCBs) with annular electrical contacts surrounding drilled holes to electrically contact each sides of the film separately. Circuit board electrodes were attached to the PVDF film using silver conductive epoxy adhesive (MG Chemicals, Model 8331). The skin-facing side of the PVDF was electrically grounded and covered in a $1 \mathrm{~mm}$ PDMS film (Ecoflex 00-10) with a similar mechanical impedance to muscle. The opposite side of the PVDF film used one of three backing materials to constrain the mechanical resonant modes and control the acoustic response. The three backings included: PDMS (Ecoflex 00- 10), silicone gel (Dow Corning SYLGARD 527 dielectric gel) plus polyimide tape, or an open backing (air-backed). Backings were applied at thicknesses of $1 \mathrm{~mm}$ (PDMS) and $1 \mathrm{~mm}$ (gel). Four sensor diameters $(2,4,8$, and $16 \mathrm{~mm})$ were also tested to study the effect of sensor size on acoustic sensitivity and frequency response. Each combination of size and backing material was replicated on 36 test boards. 

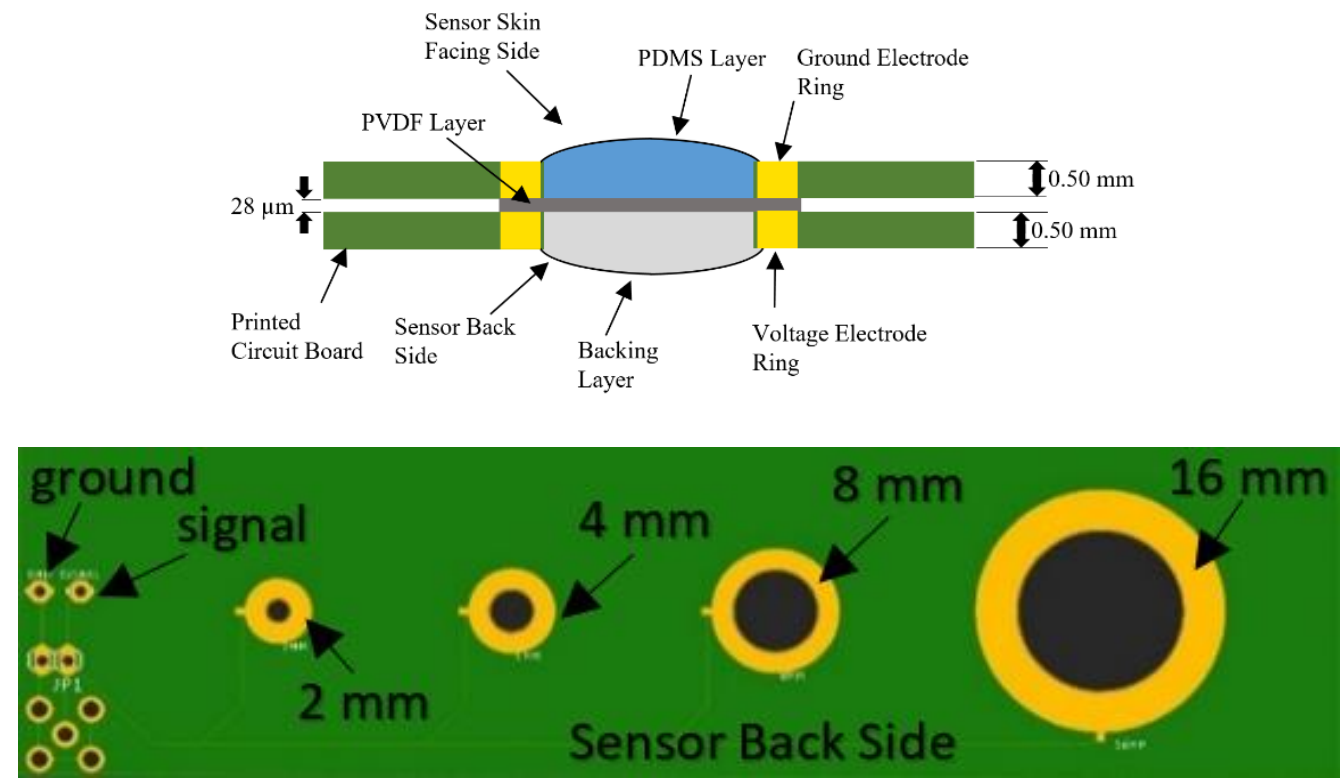

Figure 8: Cross-sectional view of the sensor showing the PVDF film sandwiched between two electrodes and a layer of PDMS on the skin-facing side. (B) Layout of a sensor test board containing $2,4,8$, and $16-\mathrm{mm}$ diaphragm sizes.

\subsection{Size and backing material characterization}

All sensors were tested under the same conditions to determine the best combination of sensor size and backing material, and their responses were compared to a conventional stethoscope. Characterization test included slow frequency sweeps to determine the acoustic frequency response and single-tone tests to determine Signal to Noise Ratio (SNR). Sensor data were collected using LabVIEW at sampling rate of $10 \mathrm{kHz}$. Sensor data were compared to the data collected from a digital recording stethoscope (3M Littmann Stethoscope 3200). The sampling rate of the stethoscope was $4 \mathrm{kHz}$, but this was digitally upsampled to $10 \mathrm{kHz}$ to match the sample rate of the PVDF sensors for frequency analysis. Output response of the speaker was measured with a contact microphone (Tyco Electronics CM-01B) with an approximately flat (i.e., broadband) response until $2 \mathrm{kHz}$. 


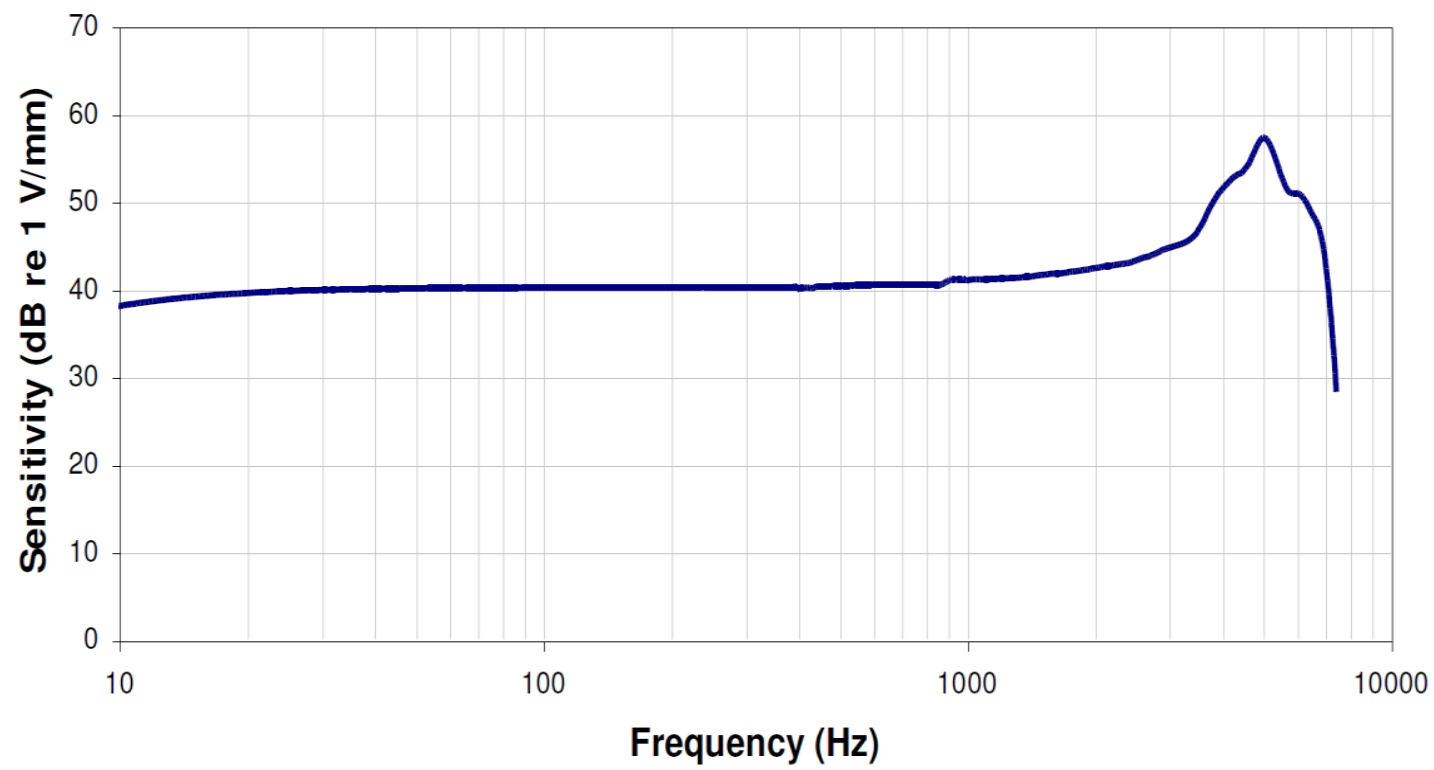

Figure 9: Typical frequency response curve for the microphone, which has a flat response until 2 $\mathrm{kHz}$.

\subsubsection{Frequency Response and Signal to Noise Ratio Calculation}

A frequency generator (Agilent 33522A, Function/Arbitrary Waveform Generator) was used to generate a logarithmic frequency sweep from $20 \mathrm{~Hz}$ to $5 \mathrm{~Hz}$ over 60 seconds. To avoid harmonics due to overdriving, the driving voltage was limited to $50 \mathrm{mVpp}$. The output was connected to a contact speaker element to generate acoustic vibrations through $6 \mathrm{~mm}$ layer of PDMS rubber (Figure 10). This arrangement mimicked the typical thickness of tissue over a blood vessel in a vascular access [50]. To account for variations in surface coupling pressure and gain differences, recording from sensors and the stethoscope were normalized to a -10 dB RMS level in MATLAB.

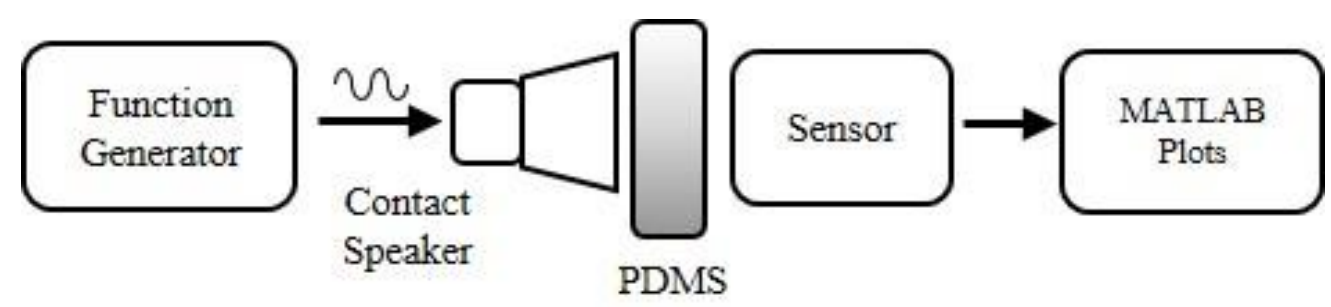

Figure 10. Test setup used for frequency response measurements. 
This test showed that all the four diameters have comparable frequency response and in order to keep the sensor small. The smallest size $(2 \mathrm{~mm})$ was chosen.

Single tone testing was carried out at 3 frequencies $(100,1000$, and $1500 \mathrm{~Hz})$ using the same setup. To compare the response between sensors and the stethoscope, the Signal to Noise Ratio (SNR) was calculated. SNR values are grouped in the basis of backing material (Figure 11) and averaged across all tested frequencies and all sensor sizes. On average, all PVDF sensors outperformed the stethoscope. The sensors with silicone gel backing performed better than other two backings. Thus, $2 \mathrm{~mm}$ sensor with silicone gel was chosen for array integration.

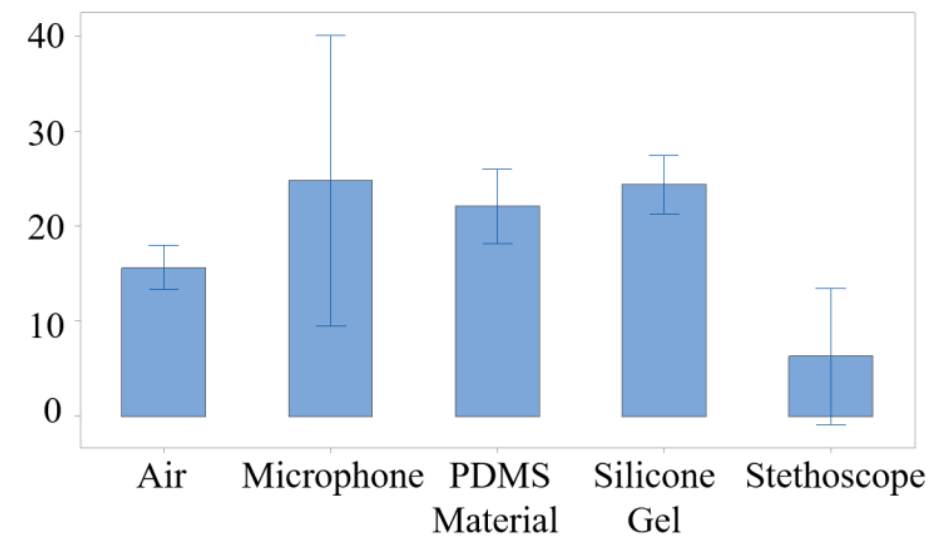

Figure 11. Sensors with silicone gel backing had the highest SNR and performed well at all the three test frequencies.

TABLE 3: MEASURED SNR OF PVDF SENSORS, MICROPHONE, AND STETHOSCOPE

\begin{tabular}{|c|c|c|c|}
\hline Backing Material & Observations & $\begin{array}{c}\text { SNR (dB) Mean } \pm \\
\text { SD }\end{array}$ & $\begin{array}{c}\text { 95\% Confidence } \\
\text { Interval for the } \\
\text { Mean }\end{array}$ \\
\hline Air & 27 & $15.66 \pm 2.97$ & $13.30-18.02$ \\
\hline Microphone & 9 & $24.86 \pm 9.95$ & $9.55-40.17$ \\
\hline PDMS & 27 & $22.16 \pm 4.97$ & $18.22-26.09$ \\
\hline Silicone Gel & 27 & $24.40 \pm 3.83$ & $21.36-27.43$ \\
\hline
\end{tabular}




\begin{tabular}{|c|c|c|c|}
\hline Stethoscope & 9 & $6.34 \pm 5.45$ & $-0.9-13.57$ \\
\hline
\end{tabular}

\subsubsection{Array of Sensors}

The $2 \mathrm{~mm}$ sensor with silicone gel was used to build an array of sensors. The main objective was to use the array to localize the stenosis and analyze the acoustic characteristics around the stenosis rather than a single point analysis. Therefore, a $1 \times 5$ array of sensors was designed which could be oriented along the direction of vascular flow. Testing PAGs from different locations around the stenosis on vascular access phantom showed that a resolution of $1 \mathrm{~cm}$ is enough to characterize different acoustic properties as the test showed recordings at different locations (up to $3 \mathrm{~cm}$ proximal and distal to the stenosis) had different features ( $\overline{A S C}$ and $A S F_{R M S}$ ) value (detailed results below). Thus, the array sensor consisted of five $2 \mathrm{~mm}$ sensor with silicone gel backing, each spaced $1 \mathrm{~cm}$ apart. Sensors were integrating using a flexible polyimide substrate with laser-cut relief between recording sites (Figure 12). The polyimide substrate with relief cuts had reduced surface area and good vibration damping, allowing recording from five locations simultaneously without any measurable crosstalk. Through an experiment it was observed that when one of the sensor locations was stimulated using a point source, the sensitivity ( $\mathrm{SNR}$ at $100 \mathrm{~Hz}$ ) at the stimulated sensor was $29.71 \pm 1.12 \mathrm{~dB}$ while the sensitivity dropped to $-11.76 \pm 4.96 \mathrm{~dB}$ at the sensor $1 \mathrm{~cm}$ away and $-23.81 \pm 4.21 \mathrm{~dB}$ at the sensor $2 \mathrm{~cm}$ away. Thus confirming low crosstalk between the sensors. This sensor array was used on vascular bench phantoms to determine the sensitivity and specificity of stenosis detection and classification using PAG-based spectral feature analysis. 


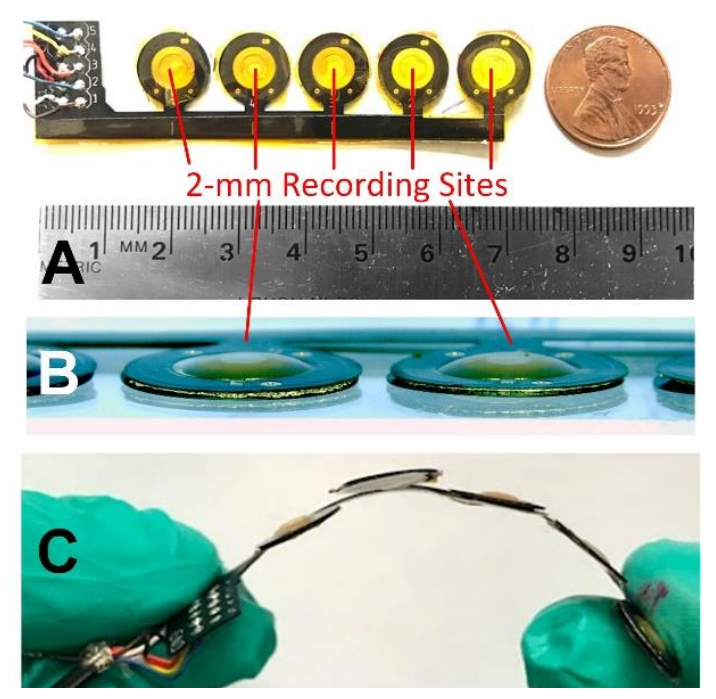

Figure 12: Prototype PAG sensor array based on 2-mm diameter PVDF microphones on polyimide substrate with silicone skin-coupling interface (A). PAG array is thinner than $1 \mathrm{~mm}$ (B) and flexible to conform to vascular access shape (C).

\subsection{Sensor Interface Amplifier Design}

In order to read signals from the sensor, we need to amplify them before feeding into LabView. We used a transimpedance amplifier for interfacing to the high-impedance PVDF materials and a multiple feedback amplifier for voltage amplification and signal band-limiting. In this section, we introduce the design and measured test results of the amplifiers.

\subsubsection{Electrical equivalent model of PVDF Sensor}

The sensor is modeled electrically as a capacitor in parallel with a resistor and a current source. We used an impedance analyzer (Hioki IM3570) to obtain the resistance and reactance at $5-5000 \mathrm{~Hz}$ from a 2-mm PVDF sensor. These values represented an equivalent sensor circuit as shown in Figure 13. Modeled component values were calculated from the resistance/reactance for an equivalent sensor model summarized in TABLE 4. 


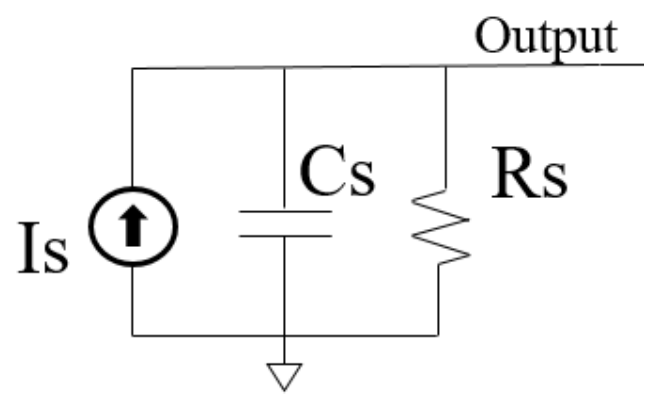

Figure 13: Electrical equivalent model of the PVDF sensor.

TABLE 4: MODEL PARAMETER VALUES OF THE PVDF SENSOR

\begin{tabular}{|l|l|}
\hline Parameter & Value \\
\hline Current Source Amplitude $\left(\mathbf{I}_{\mathbf{s}}\right)$ & $15 \mathrm{nA}-30 \mathrm{nA}$ \\
\hline Current Source Frequency & $100 \mathrm{~Hz}$ \\
\hline Sensor Capacitance $\left(\mathbf{C}_{\mathbf{s}}\right)$ & $1 \mathrm{nF}$ \\
\hline Sensor Resistance $\left(\mathbf{R}_{\mathbf{S}}\right)$ & $12.4 \mathrm{M} \Omega$ \\
\hline
\end{tabular}

\subsubsection{Transimpedance Amplifer (TIA)}

The transimpedance amplifier (TIA) is a common op amp application with an output voltage depending on the input current and a feedback resistor [51]. It is suitable for highimpedance sensors, such as the PVDF used in this application.

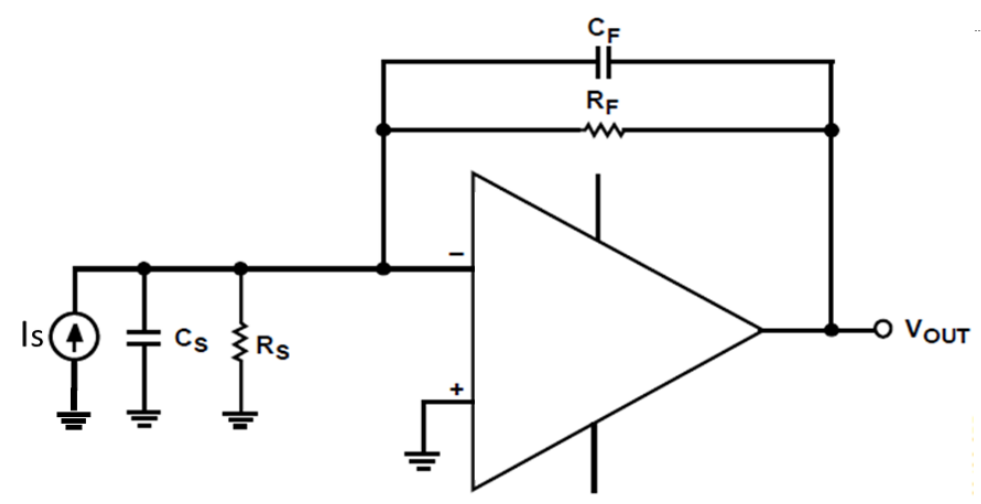

Figure 14: Circuit diagram of a transimpedance amplifier (TIA).

Design Requirements for the pre-amplifier were:

- Supply Voltage: $3.3 \mathrm{~V}$

- Input: $0 \mathrm{nA}$ to $30 \mathrm{nA}$ 
- Output: $0.1 \mathrm{mV}$ to $30 \mathrm{mV}$

- $\quad$ Bandwidth: $1.5 \mathrm{kHz}$

The transfer function between the output voltage $\left(\mathrm{V}_{\text {out }}\right)$, the input current $\left(\mathrm{I}_{\mathrm{IN}}\right)$ and the reference voltage $\left(\mathrm{V}_{\mathrm{REF}}\right)$ was defined as

$$
\begin{array}{r}
V_{\text {out }}=I_{I N} \times R_{F}+V_{R E F} \\
V_{R E F}=V_{+} \frac{R_{1} \times R_{2}}{R_{1}+R_{2}}
\end{array}
$$

To set $V_{\text {REF }}$ to midpoint level we choose $R_{1}$ and $R_{2}$ as $10 \mathrm{k} \Omega$ in eqn, (2).

For the preamplifier stage, we chose $\mathrm{V}_{\text {out }}$ to be $1.95 \mathrm{~V}$. The required feedback resistance is calculated as:

$$
R_{F}=\frac{V_{O U T}-V_{R E F}}{I_{I N}}=\frac{1.95-1.65 \mathrm{~V}}{30 \mathrm{nA}}=\frac{30 \mathrm{mV}}{30 \mathrm{nA}}=1 \mathrm{M} \Omega
$$

To calculate the feedback capacitor and the desired $-3 \mathrm{~dB}$ bandwidth, we use

$$
C_{F}=\frac{1}{2 \pi f_{-3 d B} R_{F}}=\frac{1}{2 \pi \times 1500 \times 1 M \Omega}=100 p F
$$

The minimum op amp bandwidth required for this application is based on the values of $R_{F}, C_{F}$, and the capacitance on the IN- pin of the TLV 9002 (Texas Instruments) which is equal to the sum of sensor capacitance $\left(\mathrm{C}_{S}\right)$, the common-mode input capacitance $(\mathrm{CCM})$ and the differential input capacitance $\left(\mathrm{C}_{\mathrm{D}}\right)$. Thus, it is given by

$$
\mathrm{C}_{\mathrm{IN}}=\mathrm{C}_{\mathrm{S}}+\mathrm{C}_{\mathrm{CM}}+\mathrm{C}_{\mathrm{D}}=1000 \mathrm{pF}+5 \mathrm{pF}+1 \mathrm{pF}=1006 \mathrm{pF}
$$

The minimum op amp bandwidth is calculated as 


$$
f_{=B G W} \geq \frac{C_{I N}+C_{F}}{2 \times \pi \times R_{F} \times C_{F}^{2}} \geq 17.61 k H z
$$

The $1 \mathrm{MHz}$ bandwidth of the TLV900x meets the minimum bandwidth requirement and remains stable in this application configuration [52]. Input-referred noise is calculated as

$\mathrm{i}_{\mathrm{n}}$ (Input current noise density) at $\mathrm{f}=1 \mathrm{kHz}=23 \mathrm{fA} / \sqrt{\mathrm{Hz}}$ From the datasheet

Bandwidth $=1500 \mathrm{~Hz}$

Noise: $23 \mathrm{fA}^{*} \sqrt{1500}=0.89 \mathrm{pA}_{\mathrm{rms}}$.

\subsubsection{Multiple Feedback Amplifier}

We designed a multiple feedback amplifier to provide $30 \mathrm{~dB}$ gain over a 1 - $2000 \mathrm{~Hz}$ signal bandwidth [53]. Component values were chosen from the design equation:

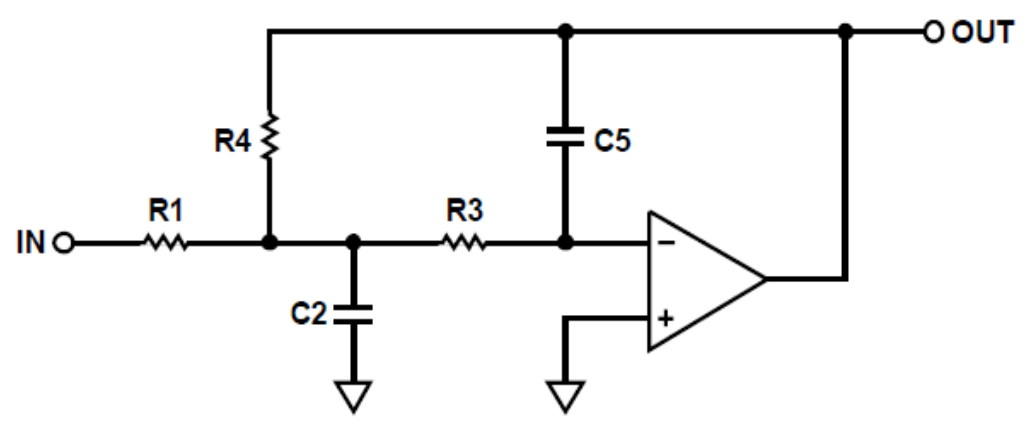

Figure 15: Circuit diagram of a multiple feedback amplifier.

$$
\begin{gathered}
\frac{V_{o}}{V_{I N}}=\frac{-H \frac{1}{R 1 R 3 C 2 C 5}}{S^{2}+s \frac{1}{C 2}\left(\frac{1}{R 1}+\frac{1}{R 3}+\frac{1}{R 4}\right)+\frac{1}{R 3 R 4 C 2 C 5}} \\
k=2 \pi f_{0} C 5
\end{gathered}
$$




$$
\begin{gathered}
C 2=\frac{4}{\alpha^{2}}(H+1) C 5 \\
R 1=\left(\frac{\alpha}{2}\right) H k \\
R 3=\frac{\alpha}{2(H+1) k} \\
R 4=\frac{\alpha}{2 k}
\end{gathered}
$$

The desired design requirements are:

- Supply voltage: $3.3 \mathrm{~V}$

- Input: $0 \mathrm{mV}$ to $30 \mathrm{mv}$

- Output: $0.1 \mathrm{~V}$ to $1 \mathrm{~V}$

- Bandwidth: $2 \mathrm{kHz}$

These are the component values that were used:

TABLE 5: VALUES OF MULTIPLE FEEDBACK AMPLIFIER COMPONENTS

\begin{tabular}{|l|l|}
\hline Parameter & Value \\
\hline R1 & $5.23 \mathrm{k} \Omega$ \\
\hline C2 & $22 \mathrm{nF}$ \\
\hline R3 & $7.5 \mathrm{k} \Omega$ \\
\hline R4 & $182 \mathrm{k} \Omega$ \\
\hline C5 & $200 \mathrm{pF}$ \\
\hline
\end{tabular}

Input-referred noise is calculated as:

$\mathrm{e}_{\mathrm{n}}$ (Input voltage noise density) at $f=1 \mathrm{kHz}$ is $30 \mathrm{nV} / \sqrt{\mathrm{Hz}}$ From the datasheet Bandwidth $=2000 \mathrm{~Hz}$ 
Noise: $30 \mathrm{nV}^{*} \sqrt{2000}=1.34 \mu \mathrm{V}$

First stage gain: $1 \mathrm{M} \Omega$

Therefore, noise referred to first stage: $1.34 \mu \mathrm{V} / 1 \mathrm{M} \Omega=1.34 \mathrm{pA}_{\mathrm{rms}}$.

Total input referred noise: $\left((0.89)^{2}+(1.34)^{2}\right)^{1 / 2}=1.61 \mathrm{pA}_{\text {rms. }}$.

\subsection{LTspice simulation and Practical Testing Values}

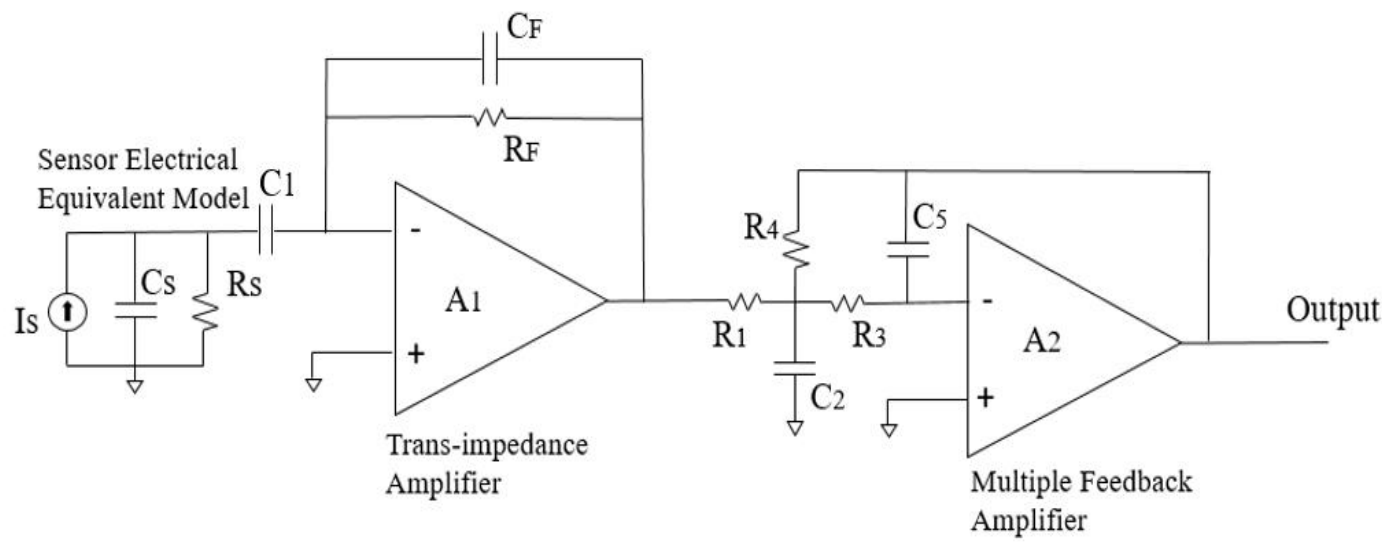

Figure 16: LTspice simulated circuit diagram.

Amplifier performance was simulated in LTspice (Figure 16), which is a widely-used free circuit simulation tool available from Analog Devices. The estimated small-signal amplifier performance is summarized in TABLE 6.

TABLE 6: PERFORMANCE OF THE SIMULATED CIRCUIT IN LTSPICE

\begin{tabular}{|l|l|l|}
\hline Parameter & Stage 1 & Stage 2 \\
\hline Input current/voltage & $0-30 \mathrm{nA}$ & $0-30 \mathrm{mV}$ \\
\hline Output voltage & $0.1-30 \mathrm{mV}$ & $0.1 \mathrm{~V}$ to $1 \mathrm{~V}$ \\
\hline Gain & $1 \mathrm{~V} / 1 \mu \mathrm{A}$ & $33 \mathrm{~dB}$ \\
\hline Bandwidth & $1.5 \mathrm{kHz}$ & $2 \mathrm{kHz}$ \\
\hline
\end{tabular}




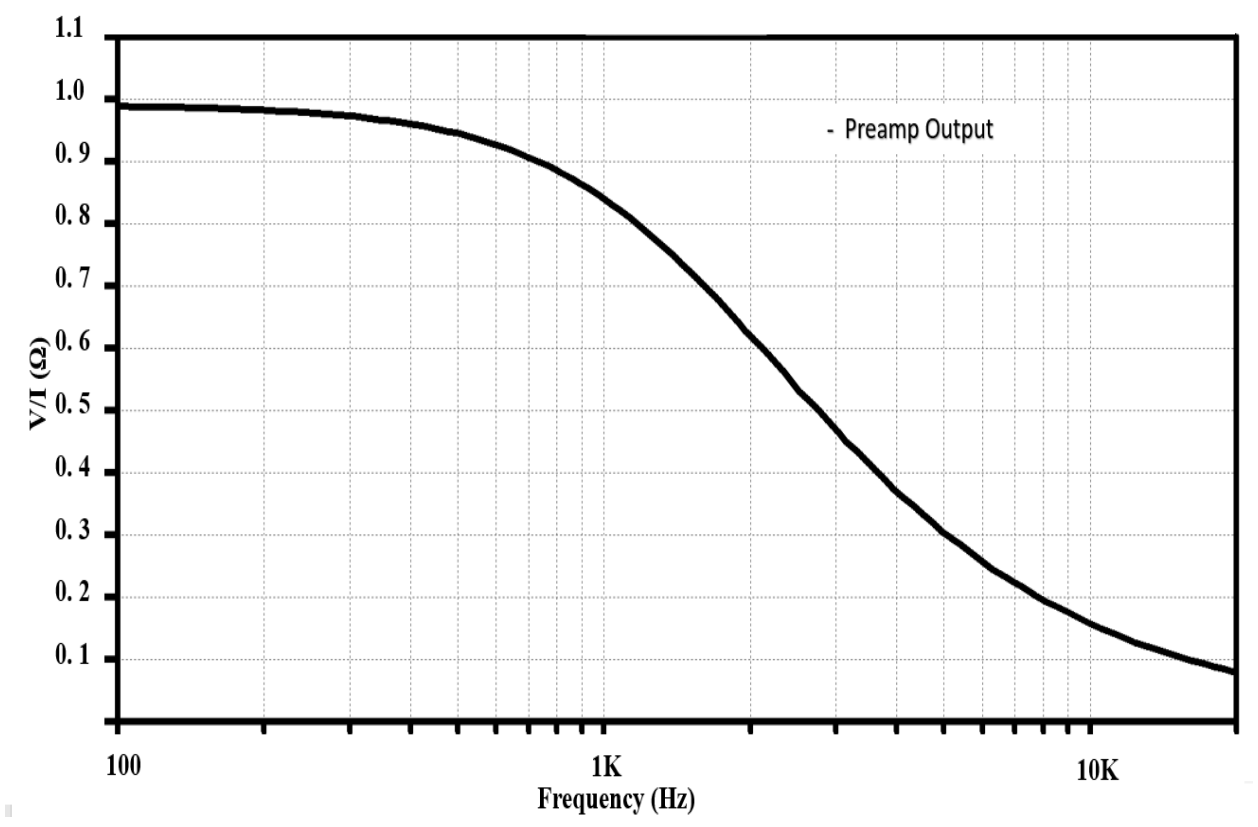

Figure 17: Simulated small-signal frequency response of the TIA.

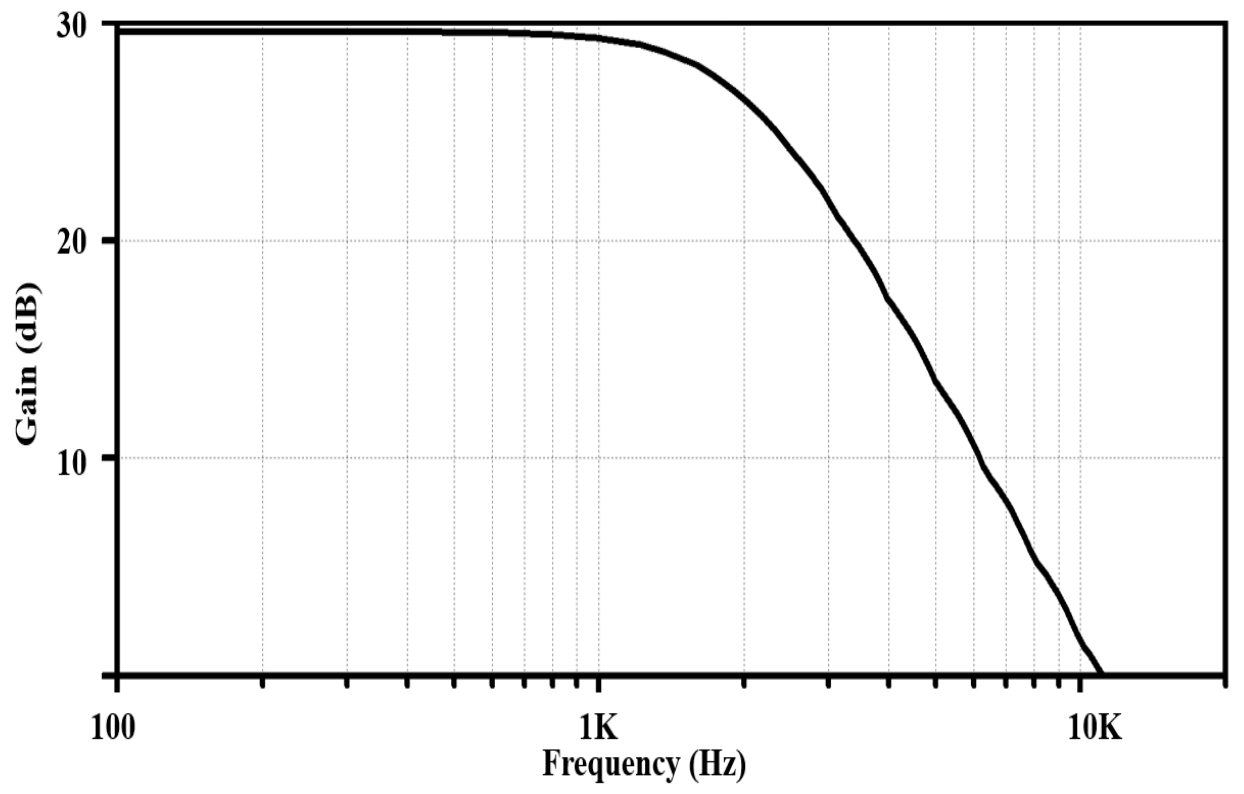

Figure 18: Simulated small-signal frequency response of the multiple feedback amplifier.

Finally, the series capacitor $\mathrm{C}_{1}$ is used as a DC block, i.e., used to prevent any DC voltage across the sensor from affecting the amplifier circuit. This is important since in general the sensor can be pre-polarized with a DC voltage to vary its sensitivity and linear range. 
To test the amplifier circuit, we used a frequency generator to generate signal at specific frequencies from $100 \mathrm{~Hz}$ to $20 \mathrm{kHz}$. The preamplifier and multiple feedback amplifiers were tested separately. The input to the preamplifier was $6.6 \mu \mathrm{A}$ (tested with Keithley multimeter DMM 6500) and the multiple feedback amplifier was $10 \mathrm{mV}$. The measured Bode magnitude plots are shown below. It is evident that the simulated and measured plots are in good agreement with each other.

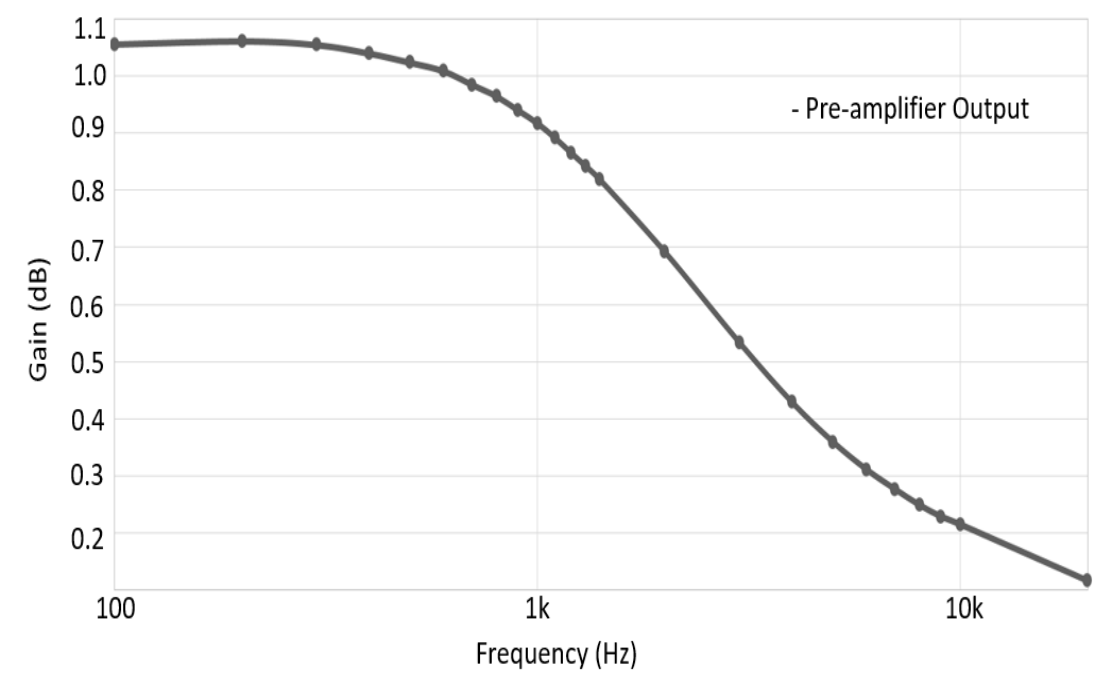

Figure 19: Measured small-signal frequency response of the TIA.

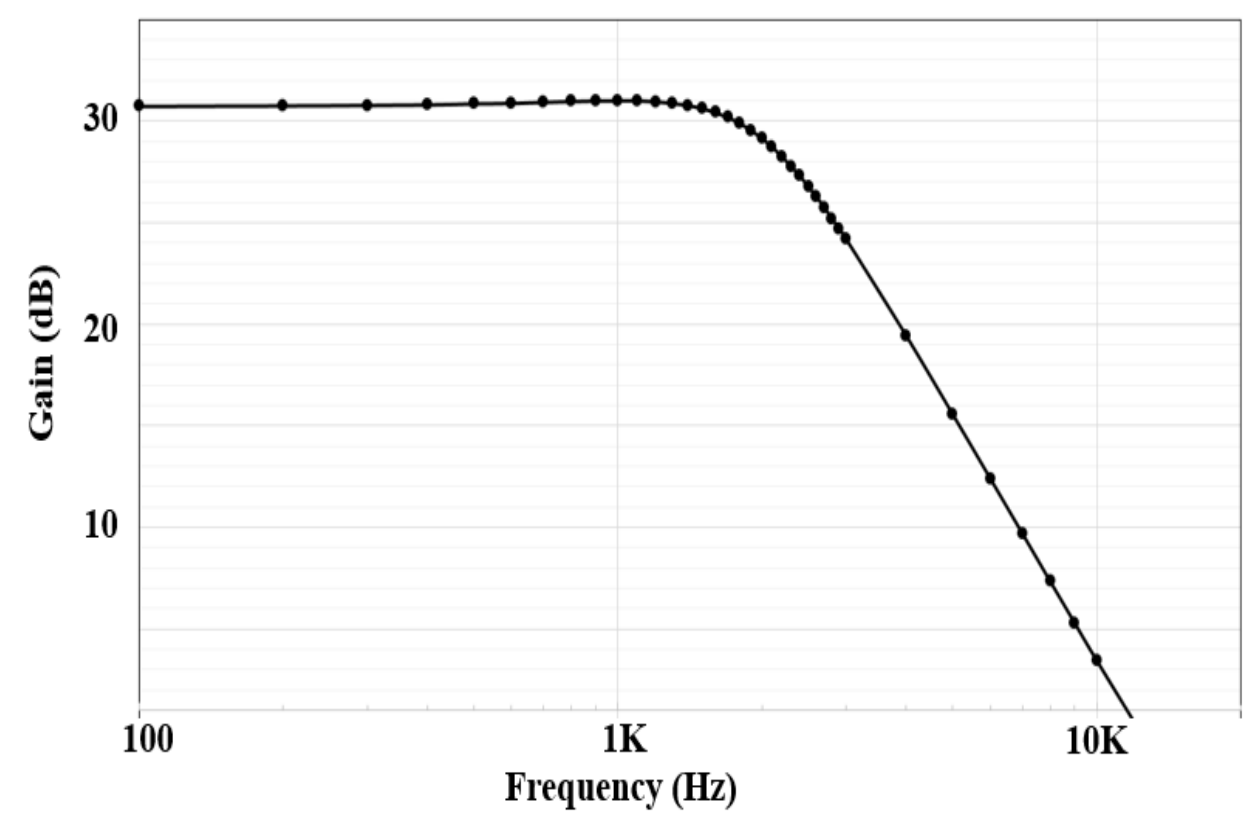
amplifier.

Figure 20. Measured small-signal circuit frequency of the multiple feedback 


\section{PHONOANGIOGRAM SIGNAL PROCESSING}

PAGs are processed in three stages, which are described separately. First, signals are preprocessed using an autoregressive bruit-enhancing filter. Next, systole and diastole phases are determined from the spectral flux. Finally, spectral features are separately calculated for systole and diastole. After features are calculated stenosis severity and location can be determined by classification methods, discussed in a later section. This section, therefore, only describes the process for extracting a set of analytical parameters from a measured PAG.

\subsection{Bruit-Enhancing Filter}

The systole portion of flow is enhanced automatically using a Bruit-Enhancing Filter (BEF) based on sub-band frequency domain linear prediction (SB-FDLP) (Figure 21). The BEF also reduces skin scratch and pop noise artifacts obtained due to movement of the recording transducer over the skin. The BEF was developed based on the nominal PAG spectra of over 1,000 bruit recordings obtained over 12 months in patients with hemodialysis vascular access (Figure 22). SBFDLP was applied on 4 bands which showed local maxima/minima in the general PAG spectra (TABLE 7) [54].

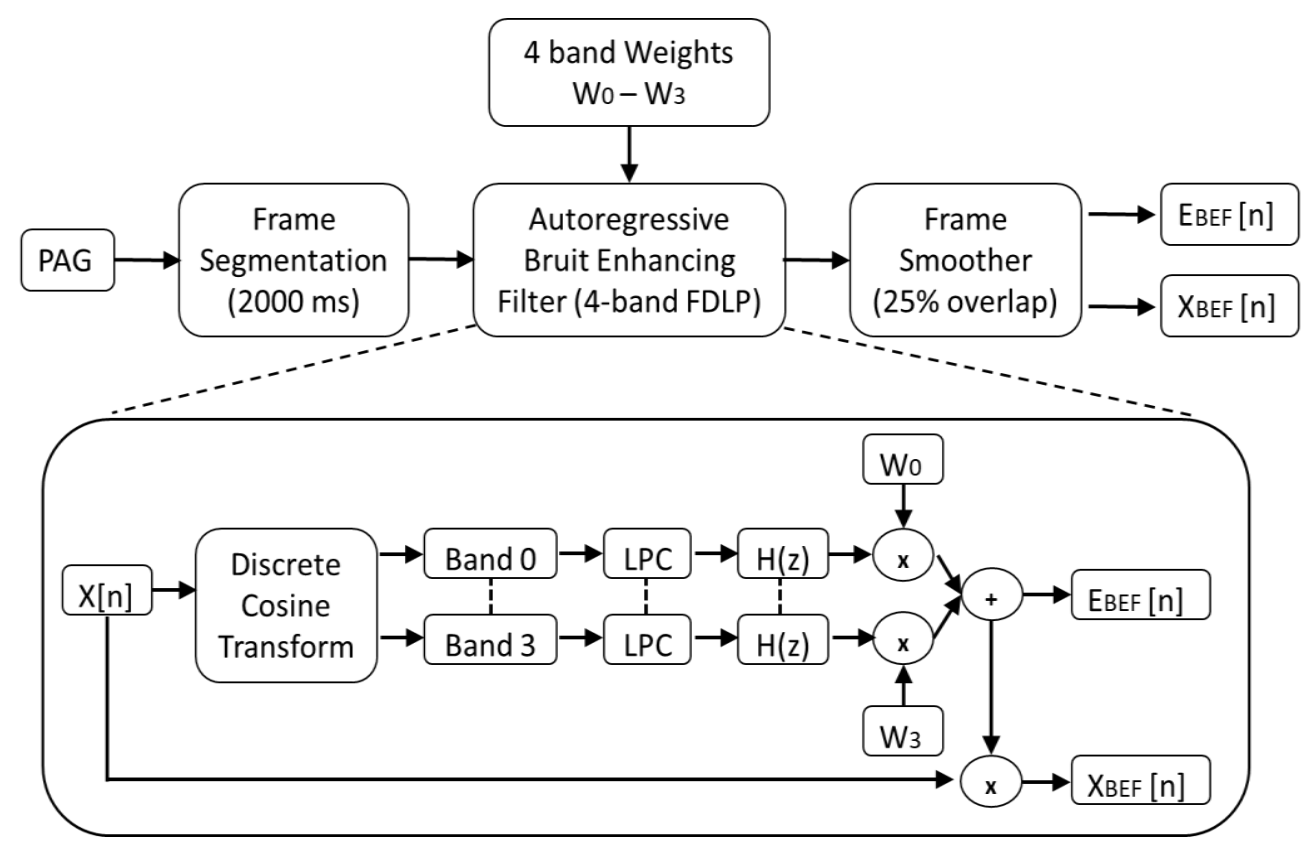


Figure 21: PAGs are processed in frames using a bruit-enhancing filter to produce an enhanced PAG $\mathrm{X}_{\mathrm{BEF}}[\mathrm{n}]$ and the bruit power envelope $\mathrm{E}_{\mathrm{BEF}}[\mathrm{n}]$. The BEF breaks the discrete cosine transform of $\mathrm{X}[\mathrm{n}]$ into $\mathrm{N}$ sub- bands. Sub-bands are modeled using linear predictive coding and recombined with weights $\mathrm{W}_{\mathrm{N}}$ to produce a bruit envelope $\mathrm{E}_{\mathrm{BEF}}[\mathrm{n}]$.

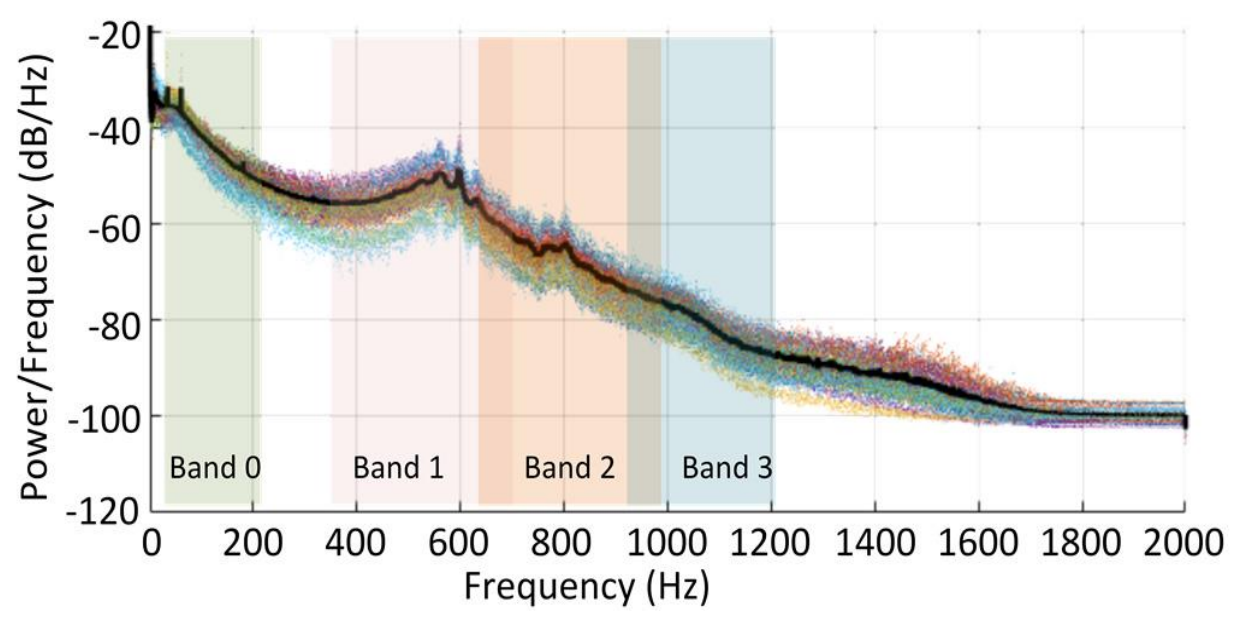

Figure 22: PAG spectra from chronic dialysis recipients (1,126 recordings). Spectra were consistent across patients over $2-12$ months of recordings. The average spectrum is (black line) revealed 4 bands with unique spectral features [54].

TABLE 7: SELECTED BANDS FOR FREQUENCY-DOMAIN MODELING

\begin{tabular}{|l|l|l|}
\hline & Sub-Band Width & Sub-Band Weight \\
\hline Band 0 & $25-225 \mathrm{~Hz}$ & $5 \%$ \\
\hline Band 1 & $350-700 \mathrm{~Hz}$ & $50 \%$ \\
\hline Band 2 & $650-1000 \mathrm{~Hz}$ & $30 \%$ \\
\hline Band 3 & $950-1200 \mathrm{~Hz}$ & $15 \%$ \\
\hline
\end{tabular}

The BEF segments the PAG into $25 \%$ overlapping frames, and models the time-domain envelope in sub-bands using SB-FDLP. Each sub-band envelope is multiplied by sub-band weights $\mathrm{W}_{0}-\mathrm{W}_{3}$ and summed to produce a modeled temporal bruit envelope $\left(\mathrm{E}_{\mathrm{BEF}}\right)$. The envelope $\mathrm{E}_{\mathrm{BEF}}$ is multiplied by the original recording to produce a bruit-enhanced PAG ( $\left.\mathrm{X}_{\mathrm{BEF}}\right)$. This approach is generalizable to other signals through the selection of sub-bands, sub-band weights, and the pole rate (number of poles used in linear prediction relative to frame length).

The core component of the BEF is frequency-domain linear prediction. When used on a broadband transform, FDLP models the Hilbert envelope of a signal [55], [56]. By controlling the 
number of poles used in the predictive model, significant smoothing of the modeled envelope is obtained. The FLDP envelopes are calculated from the real discrete-cosine transform (DCT) values of the PAG, $X_{D C T}[k, n]$ over 20,000 frequency coefficients, i.e.

$$
X_{D C T}[k]=a[k] \sum_{0}^{N-1} x[n] \cos \left(\frac{(2 n+1) \pi k}{2 N}\right),
$$

where

$$
a[k]=\left\{\begin{array}{cc}
1 & k=0 \\
\sqrt{2} & k=1,2, \ldots N-1
\end{array} .\right.
$$

The DCT approximates the envelope of the Discrete Fourier Transform (DFT). This implies that the spectrogram of the DCT (treating the DCT as a time sequence) mirrors the time-domain spectrogram around the time/frequency axes. Just like time-domain linear predictive coding (LPC) models the frequency-domain envelope, FDLP estimates the temporal envelope when applied in the frequency domain. This implementation uses LPC to model the spectral envelope using a $P^{\text {th }}-$ order, all-pole FIR filter.

$$
\hat{x}[n]=\sum_{k=1}^{p} a_{k} x[n-k], \text { i.e. } P(z)=\sum_{k=1}^{p} a_{k} z^{-k}
$$

Where $P$ is the order of the filter polynomial and $P(z)$ is its z-transform. LPC uses least-squares iterative fitting to determine the coefficients $a_{k}$ of the filter $P(z)$ such that the error in determining the next value of a series $\hat{x}[n]$ is minimized. The calculated filter is an autoregressive model with significantly lower variance than the Hilbert envelope or computed ASF when used for PAG analysis [54]. When LPC is applied in sub-bands, we refer to $P(z)$ in each individual band as $H_{m}(z)$ where $m$ is for the $m^{\text {th }}$ sub-band. Thus, we can say that the impulse response of $H_{m}(z)$, which is denoted by $H m[n]$, predicts the time-domain envelope produced by frequencies of $x[n]$ within the 
$m$ th sub-band.

The $N$ sub-band envelopes $H_{m}[n]$ are combined to produce a bruit-enhanced envelope $E_{B E F}[n]$ using sub-band weights $\mathrm{W}_{\mathrm{m}}$, resulting in

$$
E_{B E F}[n]=\sum_{m=1}^{N} W_{m} H_{m}[n]
$$

After $E_{B E F}[n]$ is calculated, it is multiplied by $\mathrm{x}[n]$ to produce the enhanced signal $\mathrm{X}_{\mathrm{BEF}}$. Because $E_{B E F}[n]$ models the systolic portions of the PAG (since that is where the frequency content in the selected sub-bands is concentrated), it can be used feature extraction or systole-diastole segmentation. Otherwise, $\mathrm{X}_{\mathrm{BEF}}$ alone may be further analyzed as described below. Figure 23A shows a PAG without the bruit-enhancing filter while Figure 23B shows the same signal with the filter. We can see that the Diastole region is calm with the filter as the power in diastole in this signal is very low.



Figure 23: Spectrogram of a PAG without the bruit-enhancing Filter (A), and with the filter (B). 


\subsection{PAG Wavelet Analysis}

After we obtain the enhanced PAG at the BEF output, continuous wavelet transform (CWT) is used to describe the spectral variance over time. Wavelet transform over $k$ scales $\boldsymbol{W}[k, n]$ was computed, e.g.,

$$
\boldsymbol{W}[k, n]=x_{P A G}[n] * \psi[n / k]
$$

where $\psi[t / k]$ is the analyzing wavelet at scale $k$. We used the complex Morlet wavelet because it has good mapping from scale to frequency, defined as

$$
\psi[n]=e^{-\left(n / 2 f_{c}\right)^{2}} e^{j 2 \pi f_{c} n}
$$

where $f_{c}$ is the wavelet center frequency. In the limit $f_{c} \rightarrow \infty$, the CWT with Morlet wavelet becomes a Fourier transform. Because of the construction of the Morlet wavelet as the wavelet $\psi[n]$ is scaled to $\psi[n / k]$, and $k$ is a factor of 2 , the wavelet center frequency will be shifted by one octave. Therefore, CWT analysis with the Morlet wavelet can be described by the number of octaves $\left(N_{O}\right)$ being analyzed (frequency span) and the number of voices per octave $N_{V}$ (divisions within each octave, i.e. frequency scales). Mathematically the set of scale factors $k$ can be expressed as

$$
\begin{gathered}
k\left[i_{O}, i_{V}\right]=2^{\left(i_{O}+i_{V} / N_{V}\right)}, \\
i_{O}=k_{0}, k_{0}+1, k_{0}+2 \ldots N_{O} \\
i_{V}=k_{0}, k_{0}+1, k_{0}+2 \ldots N_{V}
\end{gathered}
$$

where $k_{0}$ is the starting scale and defines the smallest scale value and the total number of scales $K=N_{O} N_{V}$. For PAG analysis we computed CWT with $N_{O}=6$ octaves and $N_{V}=12$ voices/octave, starting at $k_{0}=3$. After computing the CWT, pseudofrequencies $F[k]$ across all $K$ scales were 
calculated as

$$
F[k]=f_{c} / k
$$

\subsection{Wavelet-derived Auditory Signals}

Because the CWT involves time-domain convolution, each time point $n$ has a paired sequence of $k$ CWT coefficients. Therefore, the CWT can be visualized like a spectrogram plotted against the pseudofrequency axis $F[k]$. However, several temporal-spectral waveforms can also be derived to reduce the dimensionality of the CWT back to $n$. These waveforms enable simple comparison between spectral and time-domain features, enabling simplified segmentation, for example. In our work, two fundamental $n$-point waveforms are calculated from the set of CWT coefficients: Auditory Spectral Flux (ASF) and Auditory Spectral Centroid (ASC).

ASF describes the rate at which the magnitude of the auditory spectrum changes, and approximates a spectral first-order difference or first derivative. It is calculated as the variation between two adjacent time frames. It is calculated as

$$
A S F[n]=\frac{1}{K} \sqrt{\sum_{k=1}^{K}(|\boldsymbol{W}[k, n]|-|\boldsymbol{W}[k, n-1]|)^{2}}
$$

where $\boldsymbol{W}[k, n]$ is the continuous wavelet transform obtained over $\mathrm{K}$ total scales of the PAG.

In [2], the severity of Degree of Stenosis (DOS) was correlated with the sharpness of the peak in the ASF. In Figure 24, we demonstrate the spectral features that ASF describes by using an artificially-generated test waveform. The test waveform in this case is a concatenated sine signals with 6 different frequencies from 100- $1500 \mathrm{~Hz}$. We can see that the ASF curve shows a spike whenever there is a sudden change in frequency. The magnitude of the ASF waveform, therefore, describes how quickly spectral power is shifting between bands (Figure 24). This property is useful in mapping large variations in a PAG signal, such as between the systole and diastole phases. Segmentation of these phases, therefore, uses the ASF waveform (described below). 

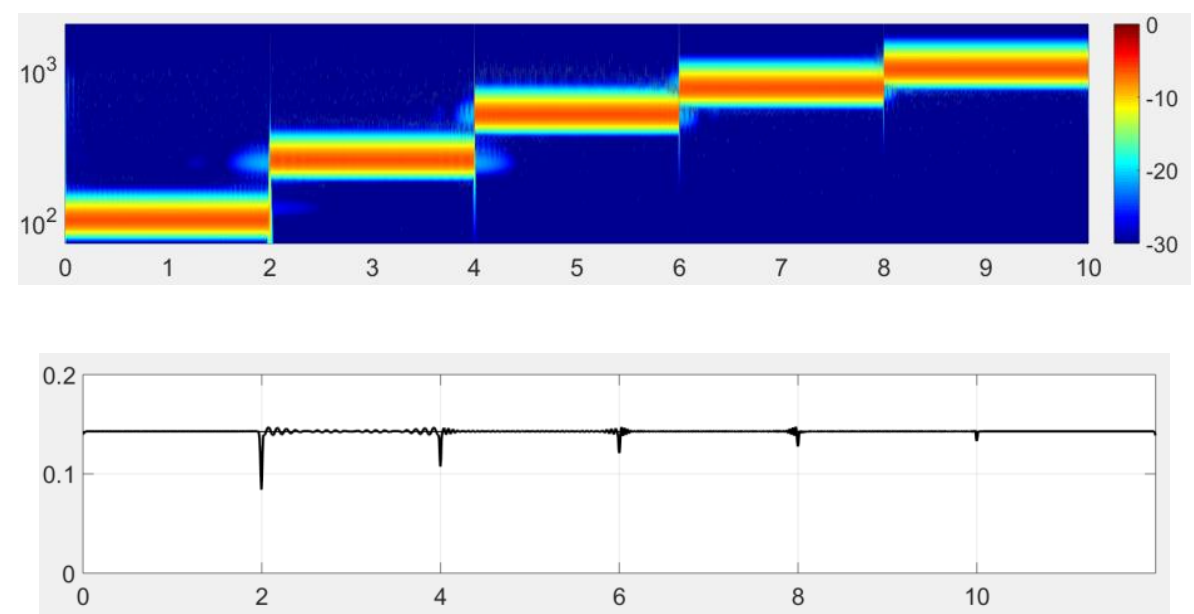

Figure 24: Spectrogram of artificially generated test waveform (top) with 6 singletone frequencies from 100-1500 Hz. The ASF curve (bottom) shows a spike at every change of frequency, approximating the spectral first derivative.

ASC describes the spectral "center of gravity" at each point in time. For Gaussiandistributed noise (same spectral power at all frequencies), ASC would be centered at pseudofrequency $F[K / 2]$. The higher value of centroid corresponds to "brighter" textures with more high frequency content [57]. ASC is commonly used to estimate the average pitch of audio recordings. It is calculated as:

$$
A S C[n]=\frac{\sum_{k=1}^{K}(|\boldsymbol{W}[k, n]| \cdot f \text { center }[k])}{\sum_{k=1}^{K}|\boldsymbol{W}[k, n]|}
$$

where $\boldsymbol{W}[k, n]$ is the continuous wavelet transform obtained over $\mathrm{K}$ total scales of the PAG and $f_{C}[\mathrm{k}]$ is the center frequency.

The spectral distribution of the bruit varies in accordance to DOS. Therefore, the severity of vascular access stenosis can be evaluated by monitoring the variation of ASC [2]. To demonstrate how ASC describes spectral content, ASC for the same test waveform was calculated (Figure 25). Because only a single tone is used at each time point, ASC consistently describes the frequency of the sine wave at each time point. As the frequency of the sine wave increases, the ASC feature also increases. Although $F[k]$ represents pseudofrequencies, the properties of the Morlet wavelet 
provide a close mapping between psuedofrequency and real frequency. For the sake of PAG analysis, absolute frequency accuracy is not needed (as discussed below).
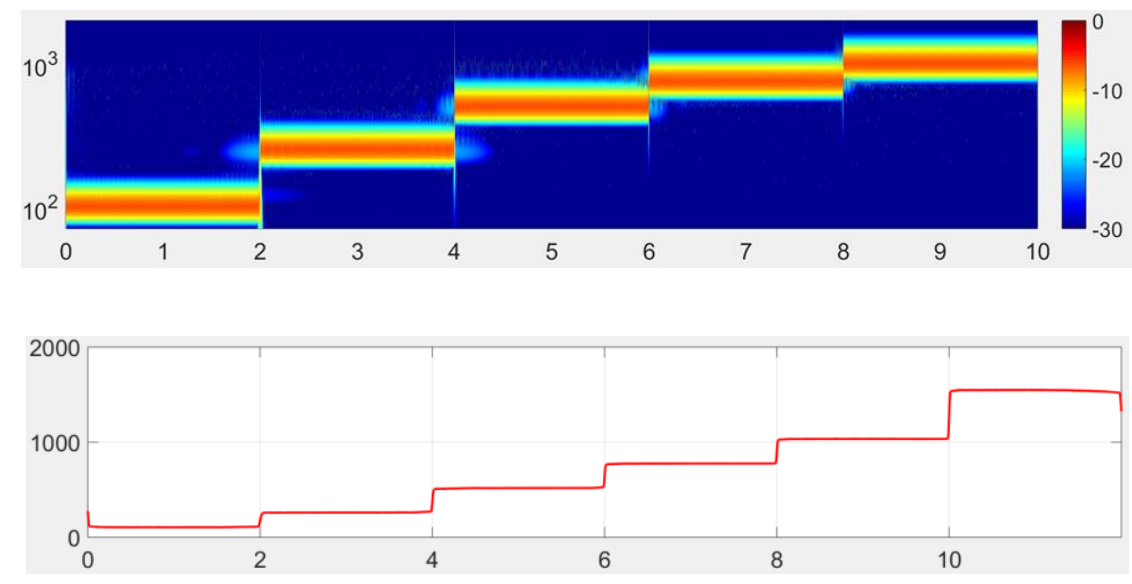

Figure 25: Spectrogram of artificially generated test waveform (top) with 6 singletone frequencies from 100-1500 Hz. The ASC curve (bottom) describes the frequency of the sine wave at each time point.

\subsection{PAG Systole/Diastole Segmentation}

During pulsatile blood flow, the high velocity flow in systole produces significant turbulence, causing the characteristic pitch of the bruit [2]. Therefore, the systolic portion of the bruit contains spectral content related to turbulent flow and should be analyzed separately from diastole to improve sensitivity. We implemented a segmentation technique to segment the pulse into systole and diastole periods. Segmentation relied on analyzing the auditory spectral flux (ASF) to identify these epochs. Firstly, 50\% of the RMS value of the ASF waveform is calculated as a threshold:

$$
V_{\text {Threshold }}=1 / 2\left(V_{\text {peak }} / \sqrt{2}\right)
$$

In particular, $50 \%$ of the RMS value of the ASF value was selected for each pulse to identify the start and end of the pulse. The pulse is width is calculated as the difference between the start and end of a pulse.

An initial identification of systole and diastole phases is performed by locating alternating threshold crossings (below threshold to above threshold for start of systole, and the opposite for 
end of systole). Then selected systolic segments were filtered through two stages. This filtering is needed to reduce threshold double-crossings or crossings caused by transient recording artifacts. In the first stage all the detected segments were considered as candidates while in the second stage, valid segments meet two conditions: maximum systolic segment length less than 1 second and greater than $40 \%$ of the longest systolic segment from stage 1 (Figure 26). This removed spurious segments caused by recording noise peaks.



Figure 26: Segmentation is performed on ASF with a 2-stage approach. Stage 1 uses threshold crossing to locate initial start and end of a segment. Stage 2 filters out spurious segments caused by recording noise peaks.

Benefits of the segmentation can be seen in an example (Figure $27 \mathrm{~A}$ ), where DOS is low (30\%) and the flow is pulsatile. The systole has more power than the diastole. While in the other case (Figure $27 \mathrm{~B}$ ), DOS is high (80\%) and the flow is turbulent. The diastole is not as calm and has a significant amount of power at frequency around $700-1000 \mathrm{~Hz}$. This is caused by turbulent flow persisting into diastole and not confined to just systole.

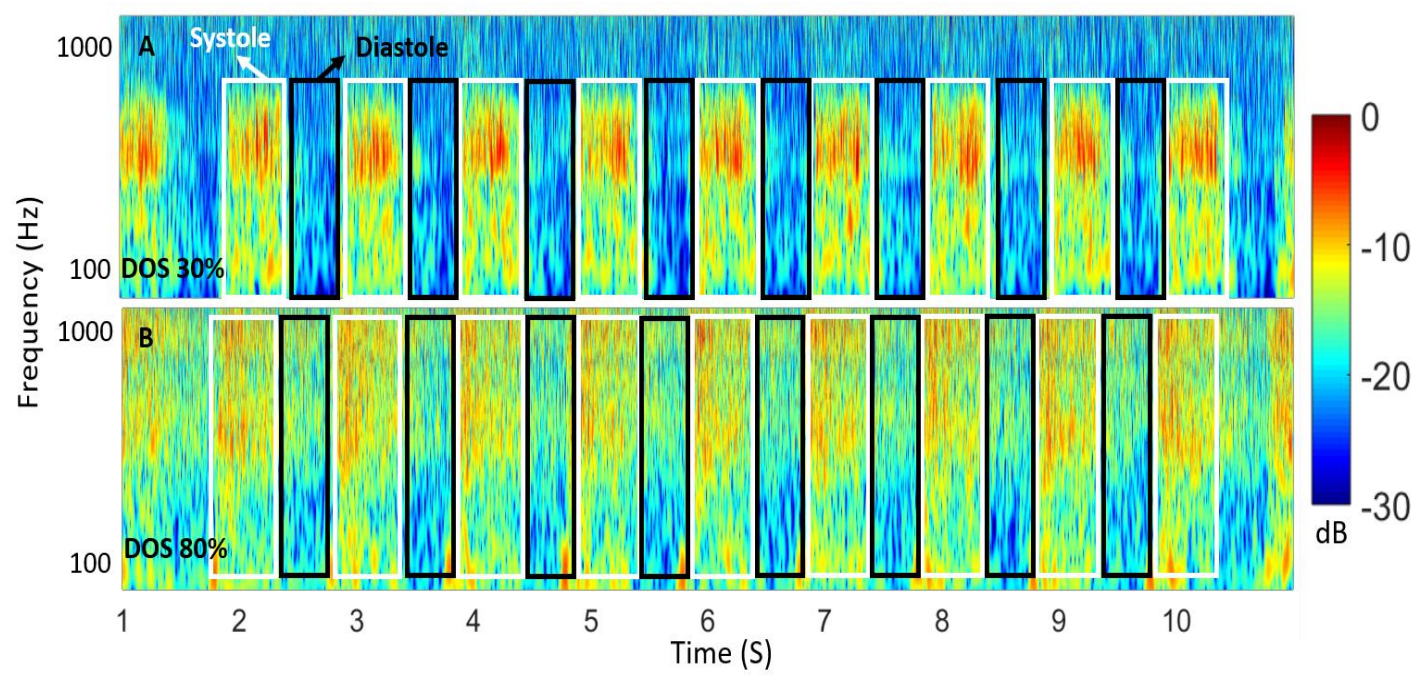


Figure 27. When degree of stenosis (DOS) is $30 \%$ (A), the power is confined to the systole phase (white box). For high DOS of $80 \%$ (B), turbulent flow increases the power in systole and diastole (black box) segments.

\subsection{PAG Spectral Feature Extraction}

We calculated 13 features for each PAG based on previous work [58]. All the features were calculated in MATLAB software (Mathworks, Natick, MA) from 10 s PAG recordings from vascular access phantoms (explained in detail in later chapters). Some features were calculated over the whole waveform, and others were calculated separately for systole and diastole phases. Our objective in feature selection was to determine features that were easily calculated and used in a simple binary classifier (discussed below). The features most sensitive to detecting DOS were determined using ANOVA and principal component analysis. Because many of the features are strongly correlated (e.g. mean and RMS), feature selection focused on features which varied the most independently. These included features 5, 6, 12, and 13 (TABLE 8). The performance of these features in a binary classification scheme was tested and is described below.

TABLE 8: LIST OF FEATURES EXPLORED

\begin{tabular}{|l|c|l|}
\hline Feature & Feature Equation & Feature Description \\
\hline 1 & $\overline{A S C}$ & Average of ASC waveform \\
\hline 2 & $\overline{A S F}$ & Average of ASF waveform \\
\hline 3 & $A S C_{R M S}$ & RMS of ASC waveform \\
\hline 4 & $A S F_{R M S}$ & RMS of ASF waveform \\
\hline 5 & $\overline{A S C_{S}}$ & Average of ASC waveform in systole segment \\
\hline 6 & $\overline{A S C_{D}}$ & Average of ASC waveform in diastole segment \\
\hline 7 & $\overline{A S C_{S}}-\overline{A S C_{D}} \mid$ & Difference in average ASC in systole and diastole \\
\hline 9 & $\overline{A S C_{S}} / \overline{A S C_{D}}$ & $\begin{array}{l}\text { Ratio of average of ASC waveform in systole and } \\
\text { diastole segments }\end{array}$ \\
\hline 10 & $A S F_{R M S, S}$ & RMS of ASF waveform in systole segment \\
\hline
\end{tabular}




\begin{tabular}{|l|l|l|}
\hline 11 & $\begin{array}{l}A S F_{R M S, S} \\
-A S F_{R M S, D}\end{array}$ & $\begin{array}{l}\text { Difference between RMS of ASF waveform in systole } \\
\text { and diastole segments }\end{array}$ \\
\hline 12 & $A S F_{R M S, S} / A S F_{R M S, D}$ & $\begin{array}{l}\text { Ratio of RMS of ASF waveform in systole and } \\
\text { diastole segments }\end{array}$ \\
\hline 13 & $\overline{A S C_{S}} * A S F_{R M S, S}$ & $\begin{array}{l}\text { Average of ASC waveform in systole segment } \\
\text { multiplied with RMS of ASF waveform in systole } \\
\text { segment }\end{array}$ \\
\hline
\end{tabular}




\section{DETECTION OF VASCULAR ACCESS STENOSIS LOCATION AND SEVERITY IN VITRO}

To simulate how the sensor would perform on a real patient (but in a controlled environment), recordings were made on vascular access phantom. The phantom consisted of a $6 \mathrm{~mm}$ silicone tube embedded in PDMS (Ecoflex 00-10) at a $6 \mathrm{~mm}$ depth. Stenosis was simulated in the center of the phantom with a band tied around the tube to produce an abrupt narrowing. The degree of stenosis (DOS) was controlled by tying the band around metal rods with fixed diameters. DOS was later confirmed by calculating the percentage of lumen diameter reduction from CT scans of phantoms (Figure 28).
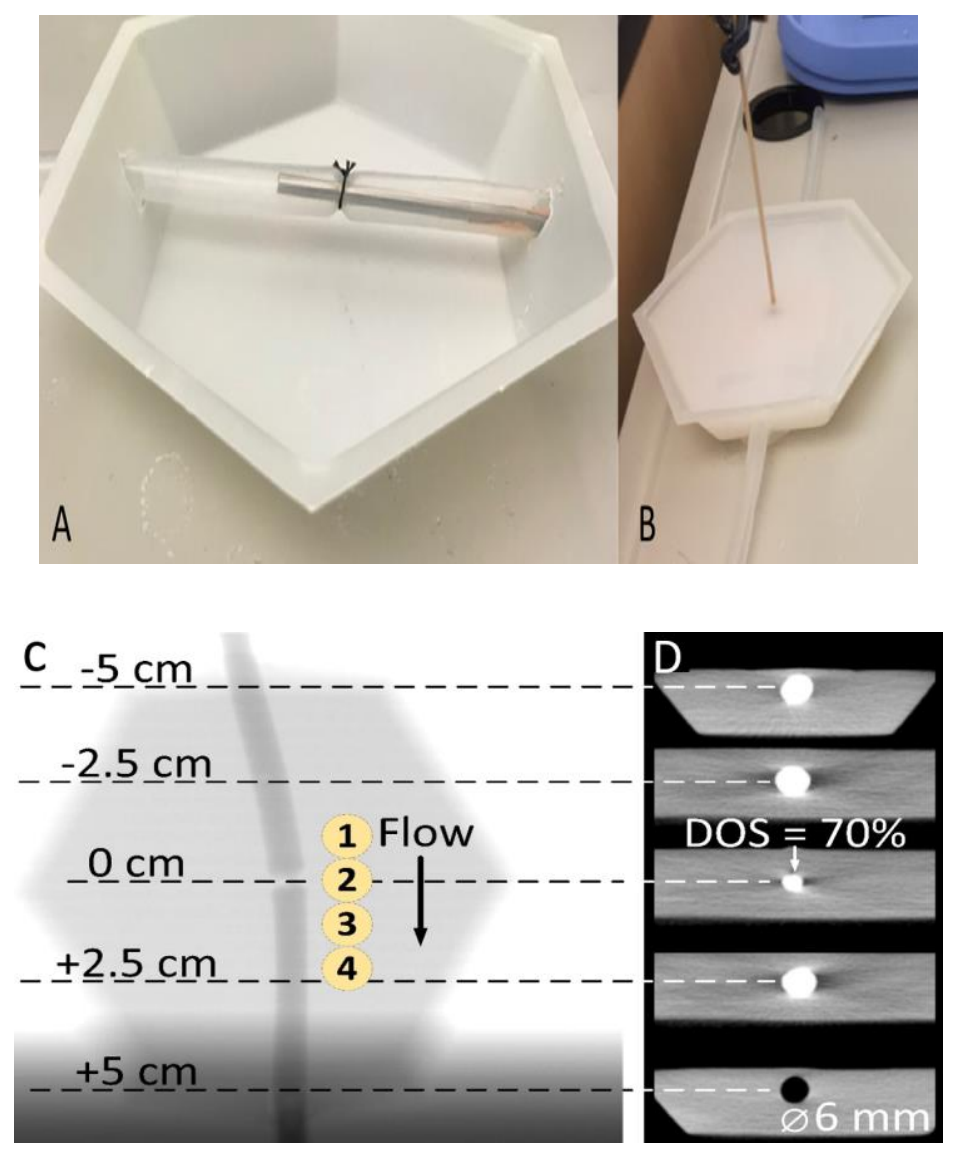

Figure 28. 10-85\% stenosis AVG phantoms used to reproduce bruits were constructed using banded 6-mm silicone tubing (A) embedded in tissue-mimicking silicone rubber to a depth of $6 \mathrm{~mm}(\mathrm{~B})$. Contrast angiography validated recording site locations (C), with computed CT slices used to calculate the degree of stenosis (D). 
The phantom was connected to a pulsatile flow pumping system (Cole Parmer MasterFlex L/S, Shurflo 4008) to simulate human hemodynamics flows from $600-1200 \mathrm{~mL} / \mathrm{min}$. Pulsatile pressures and aggregate flow rate were measured with a pressure sensor (PendoTech N-038 PressureMAT) and flow sensor (Omega FMG91-PVDF) respectively.

To validate the phantoms auditory signals, recordings were made using a digital stethoscope (Littman 3200) first and were compared to those from human to ensure that they were physiologically relevant. An aggregate power spectrum was produced from 3,283 unique 10-s recordings obtained from 24 hemodialysis patients over 18 months [54]. Spectral comparisons between human and phantom data were used to reduce time-domain variability e.g. due to heart rate differences. Human and phantom spectra followed similar spectral trends and showed similar dynamic range in time-domain analysis. Quantitatively, normalized RMS error of the aggregate power spectrum was calculated over the aggregate human PAG spectra range (Figure 22). The vascular phantoms matched the aggregate human PAG spectra with a $1.84 \%$ normalized RMS error, scaled to the total power spectrum range. This comparison suggested that the vascular stenosis phantoms can adequately replicate PAGs as measured from humans, but with control over vascular access flow rate and stenosis severity [58].

We investigated PAGs from 10 phantoms with degree of stenosis from 10-85\%. 10 second recordings were recorded with the flexible sensor array (discussed earlier) at 6 flow rates (700 $1200 \mathrm{~mL} / \mathrm{min}$ ) to represent nominal physiological flow rate in functional vascular accesses [58]. PAGs were recorded at four locations simultaneously, with one being proximal, one on the stenosis and two other distal to stenosis (Figure 28) with LabView software and National Instruments data acquisition hardware. After recording, signals were transferred to MATLAB for signal processing and calculating the features based on ASF and ASC. Signals were segmented into systole and diastole phases and features calculated separately. Because the ASF has a significant pulsatile 
component, RMS ASF values were used, while otherwise mean ASC values were calculated in each pulse phase.

ASC and ASF values were analyzed with two primary outcomes: localization of the stenosis, and differentiation of the degree of stenosis. Balanced Analysis of Variance (ANOVA) was conducted to detect differences in PAGs at different locations and at different degree of stenosis over a range of flows. The effect of flow on ASC and ASF was not studied; variable flow was used to represent this confounding variable in humans [2]. ANOVA was used to test the differences between means for statistical significance. The total number of recordings were 50 per location including all phantoms and flow rates. After segmentation there were approximately 350 systole and diastole segments for each phantom-location combination in this analysis.

\subsection{Performance of the Measured Features}

\subsubsection{Auditory Spectral Flux (ASF)}

Systole ASF RMS $\left(A S F_{R M S, S}\right)$ was different at all recording locations $(p<0.033$ for locations $1-$ 3 and $1-2$, and $p<0.001$ for all others) (Figure 29). Because array sites were located $1 \mathrm{~cm}$ apart, this result confirms that multiple recording locations are needed for accurate detection of stenosis location. Based on this analysis, a sharp drop in $A S F_{R M S, S}$ suggests the presence of stenosis within a distance of $1 \mathrm{~cm}$ proximal to the direction of flow.




Figure 29: The flexible PVDF sensor array was tested on $10-85 \%$ stenosis phantoms with variable flow. PAG systole spectral flux RMS (regardless of DOS or flow) was different at each recording site. A sharp drop in $A S F_{R M S, S}$ at Location 2 suggests the presence of stenosis within a distance of $1 \mathrm{~cm}$ proximal to the direction of the flow.

As the DOS increased, the $A S F_{R M S, S}$ value also increased [2]. This trend can be very well seen while moving from mild to moderate to severe, there is a monotonic increase in the $A S F_{R M S, S}$ irrespective of the location (Figure 30). Measured results showed a mean ASF shift of $0.059 \pm$ 0.0035 between minor and moderate DOS and a mean ASF shift of $0.143 \pm 0.004$ between moderate and severe DOS $(n=422, p<0.001)$. This result suggests that simple threshold-based detection can be used for classification of stenosis severity.

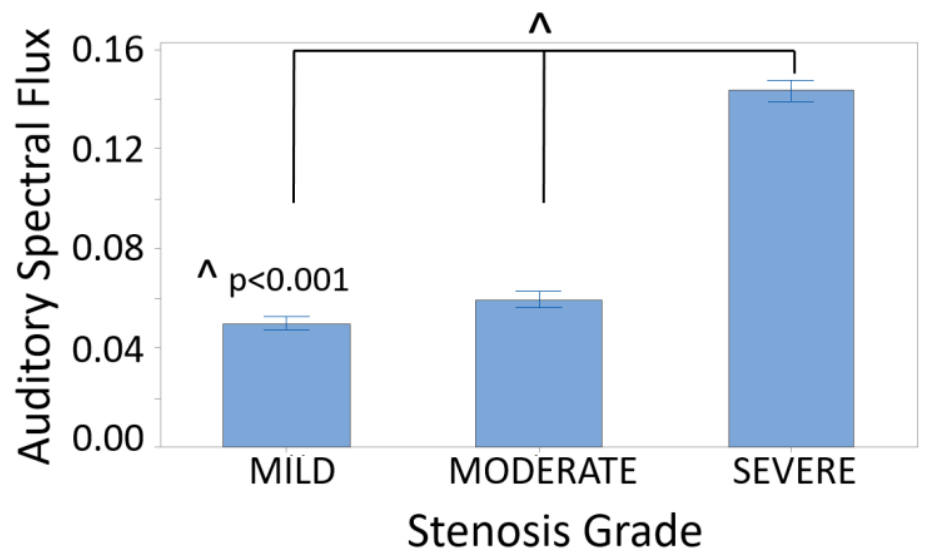

Figure 30: As the Degree of stenosis increased, the $A S F_{R M S, S}$ value also increased. This trend can be seen while moving from mild to moderate to severe DOS: there is a monotonic increase in the $A S F_{R M S, S}$ irrespective of the location.

\subsubsection{Auditory Spectral Centroid (ASC)}

Systole ASC mean $\left(\overline{A S C_{S}}\right)$ showed a similar dependence on recording location for stenosis localization. ANOVA revealed that there is a significant difference between the mean of PAGs from different locations ( $p=0.022$ for locations $2-3, p<0.001$ for all others)(Figure 31). An interesting observation was that the $\overline{A S C_{S}}$ decreased significantly (from $323 \mathrm{~Hz}$ to $223 \mathrm{~Hz}$ ) from location 3 to location 4 . This can be explained by the formation of vortex flow at areas distal to 
stenosis [59]. Prior work estimated that vortex flow occurs at a distance of 1-3 times the vessel diameter from the stenosis. In the tested phantoms, the vortex was observed at $2 \mathrm{~cm}$ from the stenosis which is 3.3 times the vessel diameter [60]. Therefore, the large drop in ASC between these locations is likely due to turbulent flow as determined by prior work. [48], [49].

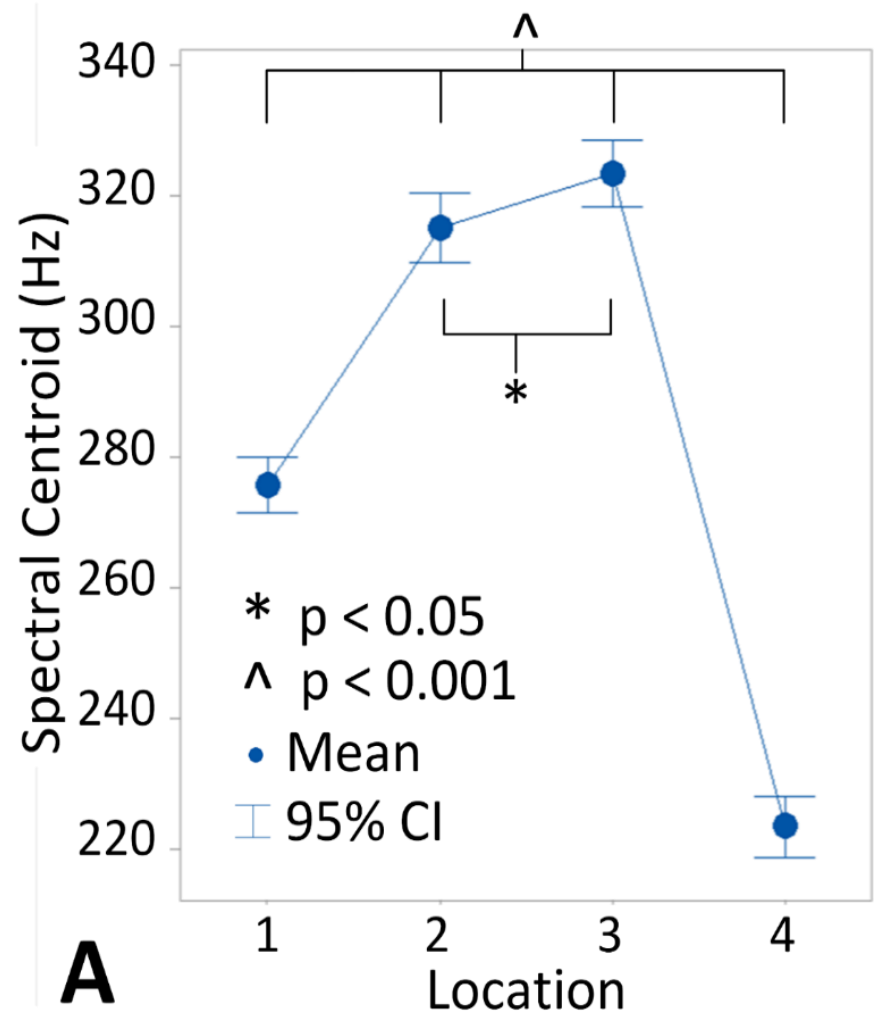

Figure 31: The flexible PVDF sensor array was tested on $10-85 \%$ stenosis phantoms with variable flow. PAG spectra (regardless of DOS or flow) (A) had distinct auditory spectral centroid (ASC) values at $1 \mathrm{~cm}$ spacings both proximal and distal to stenosis.

To find a trend in the degree of stenosis, we subtracted Systole ASC mean $\left(\left(\overline{A S C_{S}}\right)\right.$ from Diastole ASC mean $\left(\left(\overline{A S C_{D}}\right)\right.$. As DOS increases the flow becomes turbulent and power is present in both the systole and diastole segment. Thus, the difference between the mean power level is low. Since, in a low DOS phantom, most of the power is present in the systole segment, there is a huge difference between the mean power level in systole and diastole. In Figure 32A, DOS is 30\% and 
the difference between the peak in systole and valley in diastole is greater than $500 \mathrm{~Hz}$. While, in Figure 32B, this difference is less than $500 \mathrm{~Hz}$. Therefore, as DOS increases, the variation of ASC between systole and diastole diminishes and converges toward the mean ASC value of the total waveform.

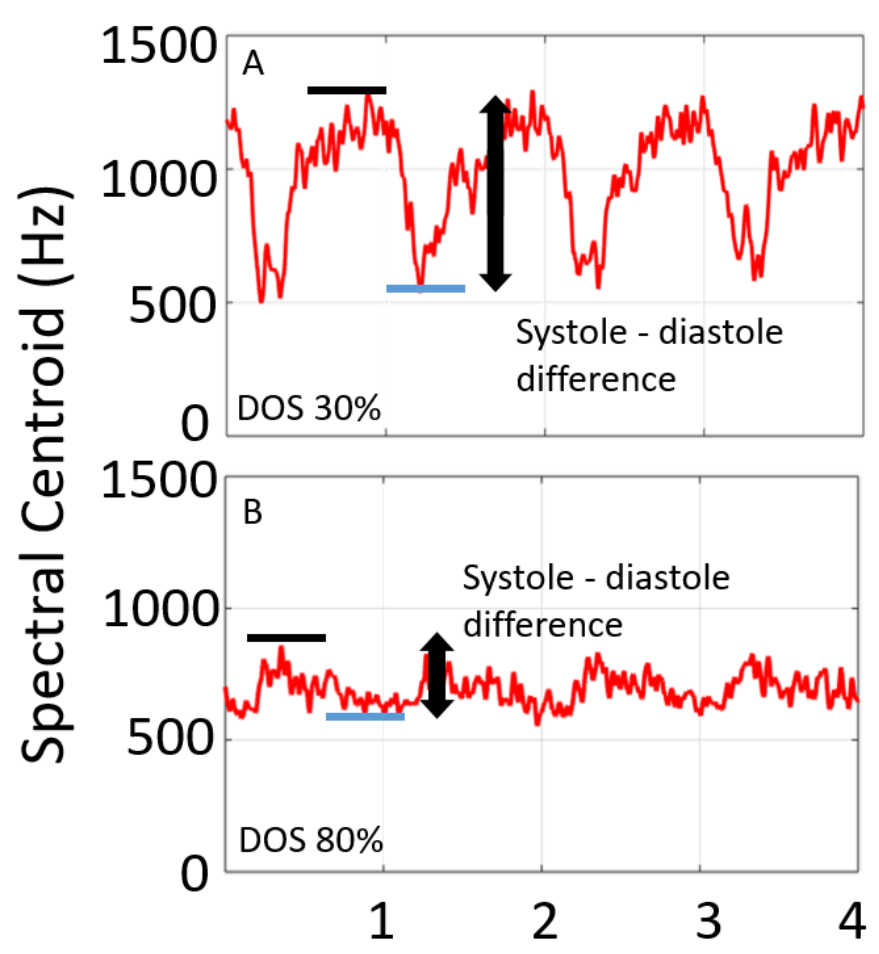

Figure 32: Low DOS (A) showed a greater peak-valley change in auditory spectral centroid (ASC) compared to high DOS (B). The difference in systole and diastole is computed after ASF-based segmentation.

The converging behavior of ASC between systole and diastole was evaluated using ANOVA. Mean systole-diastole $95 \%$ confidence intervals were $56.22 \pm 2.35$ in mild (10-40\% DOS), 44.43 \pm 3.53 in moderate $(40-60 \%$ DOS) and $14.41 \pm 2.65$ in severe (>60\% DOS) (Figure). Thus, the difference between the systole and diastole ASC values $\left(\left(\mid \overline{A S C_{S}}-\left(\overline{A S C_{D}} \mid\right)\right.\right.$ correlated strongly with stenosis grade. Tukey pairwise comparisons revealed that there was a significant difference between all stenosis grades $(p<0.001)$ regardless of stenosis grade. 


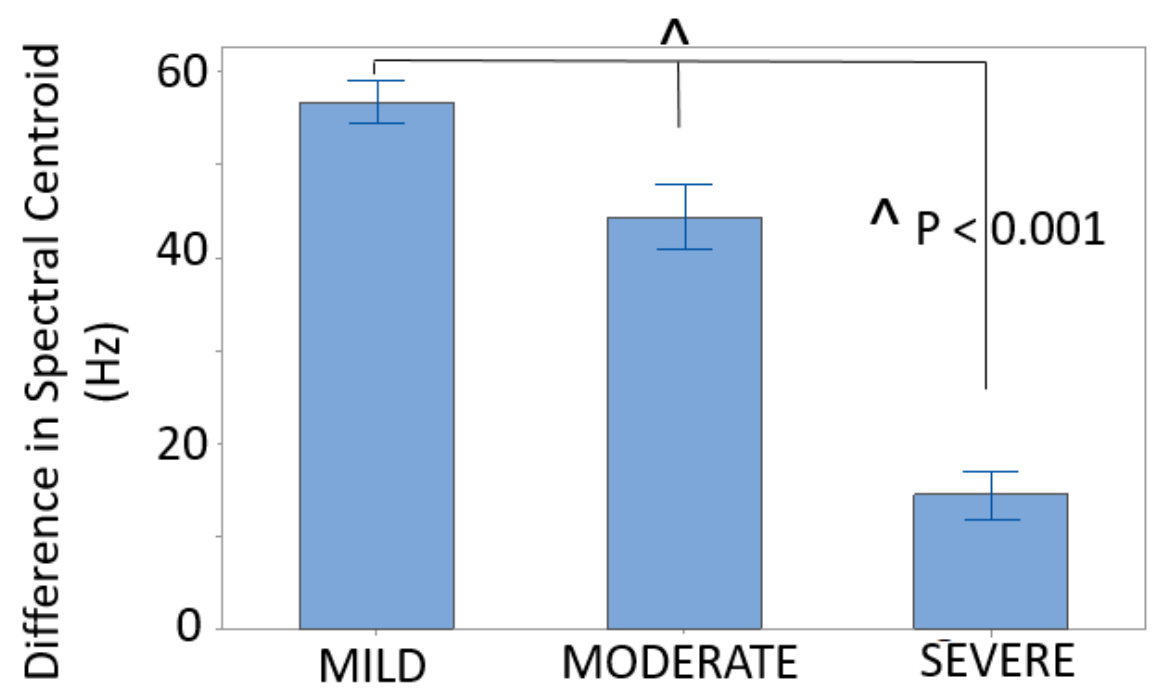

Figure 33. The difference between Systole ASC mean and Diastole ASC mean is inversely related to the DOS. As the DOS increases, the flow becomes turbulent and the difference between Systole and Diastole ASC mean decreases.

\subsection{Threshold-based phonoangiographic detection of vascular access stenosis}

Based on ASC and ASF values, we designed a binary classifier to classify DOS into two groups (TABLE 9). This classifier would be useful to identify patients with a hemodynamically-significant stenosis, defined clinically as DOS $>50 \%$. Such patients might be selected for an imaging study or entered into a vascular surveillance program to reduce emergency interventions or for treatment planning.

TABLE 9: CLASSIFIER VECTORS

\begin{tabular}{|l|l|l|}
\hline Degree of Stenosis & Signal Type & Target Vector Output \\
\hline Under 50\% & Normal (Healthy) & 0 \\
\hline Above 50\% & Pathological (Unhealthy) & 1 \\
\hline
\end{tabular}

The following performance parameters were used to measure the performance of the classifier:

- True Positive (TP): Phantom with DOS > 50\% detected correctly

- True Negative (TN): Phantom with DOS < 50\% rejected

- False Positive (FP): Phantom with DOS < 50\% detected incorrectly 
- False Negative (FN): Phantom with DOS > 50\% rejected incorrectly

Accuracy: defined as the measure of percentage of correctly classified DOS by the total number of cases

$$
\text { Accuracy }=\frac{T P+T N}{\text { Total Cases }}
$$

Specificity: defined as the measure of percentage of DOS $<50 \%$ that are recognized as DOS $<50 \%$.

$$
\text { Specificity }=\frac{T N}{T N+F P} \text {. }
$$

Sensitivity: defined as the measure of percentage of DOS $>50 \%$ that are recognized as DOS $>50 \%$.

$$
\text { Sensitivity }=\frac{T P}{T P+F N}
$$

We investigated four main features using binary classifier: Systole ASC mean $\left(\overline{A S C_{S}}\right)$, Systole $\operatorname{ASF} \operatorname{RMS}\left(A S F_{R M S, S}\right)$, difference between systole ASC and diastole ASC means $\left(\left|\overline{A S C_{S}}-\overline{A S C_{D}}\right|\right)$, and multiplication of systole ASC mean and systole ASF RMS $\left(\overline{A S C_{S}} * A S F_{R M S, S}\right)$. These features were selected from the larger set described above, based on maximum sensitivity to DOS. Each classifier was tested independently for each recording location. Classifier performance was measured by its receiver operating characteristic (ROC) curve [61]. The threshold was calculated by maximizing Youden's Index $(J)$. Youden's Index is a function of sensitivity $(q)$ and specificity (p) and is a commonly used measure of overall diagnostic effectiveness [62][63].

$$
J=\operatorname{Maximimum}\{\text { sensitivity }(c)+\operatorname{specificity~}(c)-1\}
$$

Over all threshold points $c$. The optimum threshold, $J_{O}$ was calculated for each classifier and for each location (Table 10 - Table 13). 
Table 10: PERFORMANCE OF SYSTOLE ASC MEAN FEATURE

\begin{tabular}{|l|l|l|l|l|l|}
\hline Feature & Location & $\boldsymbol{J}_{\boldsymbol{O}}$ & Accuracy & Specificity & Sensitivity \\
\hline \multirow{4}{*}{$\overline{A S C_{S}}$} & 1 & 323 & 70 & 100 & 40 \\
\cline { 2 - 6 } & 2 & 275 & 82 & 64 & 100 \\
\cline { 2 - 6 } & 3 & 312 & 78 & 80 & 76 \\
\cline { 2 - 6 } & 4 & 222 & 82 & 96 & 68 \\
\hline
\end{tabular}

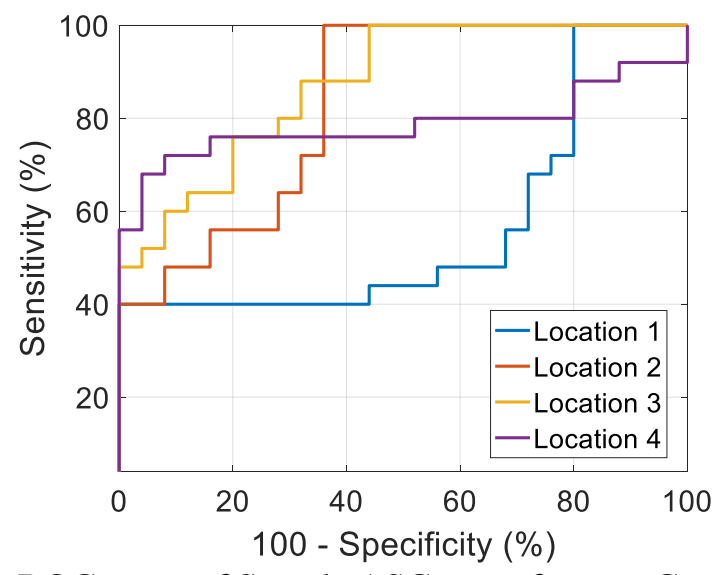

Figure 34: ROC curve of Systole ASC mean feature. Greatest area-under-the curve is achieved at Location 3 just distal to the stenosis.

Table 11: PERFORMANCE OF ASC DIFFERENCE BETWEEN SYSTOLE AND DIASTOLE

\begin{tabular}{|l|l|l|l|l|l|}
\hline Feature & Location & $\boldsymbol{J}_{\boldsymbol{O}}$ & Accuracy & Specificity & Sensitivity \\
\hline \multirow{3}{*}{$\overline{A S C_{S}}-$} & 1 & 61 & 66 & 64 & 68 \\
\cline { 2 - 6 } & 2 & 26 & 78 & 68 & 88 \\
\cline { 2 - 6 } & 3 & 56 & 84 & 76 & 92 \\
\cline { 2 - 6 } & 4 & 18 & 82 & 72 & 92 \\
\hline
\end{tabular}

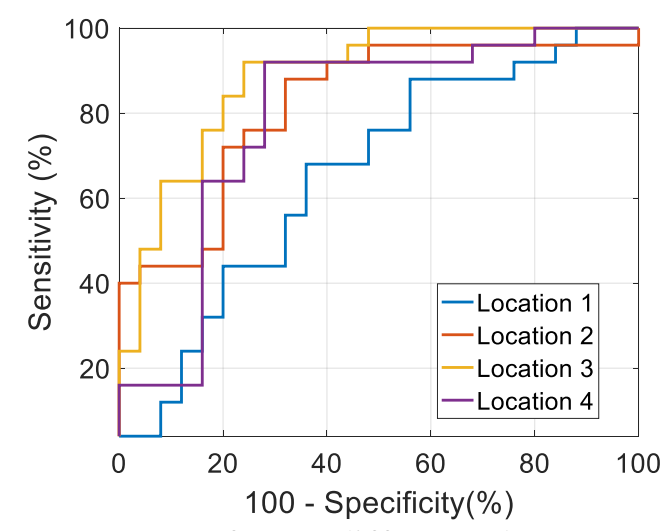

Figure 35: ROC curve of ASC difference between systole and diastole. Greatest area-under-the curve is achieved at Location 3 just distal to the stenosis.

The $\overline{A S C_{S}}$ feature had good sensitivity at Location 2 and a good specificity at Location 4. 
Location 2 is recorded directly over the point of stenosis and Location 4 is at the turbulent flow vortex. A similar trend was seen for $\left|\overline{A S C_{S}}-\overline{A S C_{D}}\right|$ feature. Overall, both the ASC features performed well with high sensitivity (68-92\%). Figure 34 and Figure 35 shows the ROC curve for these features respectively.

Table 12: PERFORMANCE OF SYSTOLE ASF RMS

\begin{tabular}{|l|l|l|l|l|l|}
\hline Feature & Location & $\boldsymbol{J}_{\boldsymbol{O}}$ & Accuracy & Specificity & Sensitivity \\
\hline \multirow{3}{*}{$A S F_{R M S, S}$} & 1 & 0.08 & 80 & 80 & 80 \\
\cline { 2 - 6 } & 2 & 0.117 & 80 & 100 & 60 \\
\cline { 2 - 6 } & 3 & 0.134 & 78 & 96 & 60 \\
\cline { 2 - 6 } & 4 & 0.088 & 94 & 100 & 88 \\
\hline
\end{tabular}

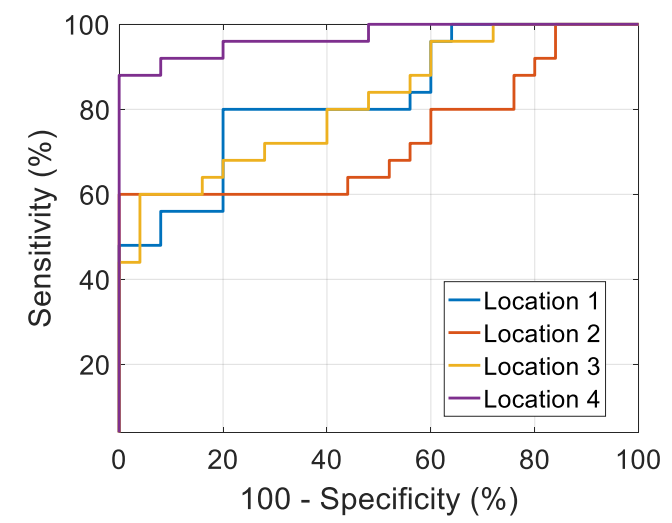

Figure 36: ROC curve of systole ASF RMS. Greatest area-under-the curve is achieved at Location 4 distal to the stenosis.

The $A S F_{R M S, S}$ feature had good specificity at locations distal to stenosis (Figure 36). Sensitivity and specificity are generally inversely proportional in nature, i.e., when sensitivity increases, the specificity decreases [64]. Thus to improve sensitivity and specificity we multiplied $\overline{A S C_{S}}$ (high sensitivity) with $A S F_{R M S, S}$ (high specificity), and achieved good overall sensitivity and specificity at Location 4 . Thus, we can use this combined feature to classify DOS $>50 \%$ with an accuracy of $90 \%$, specificity of $92 \%$ and sensitivity of $92 \%$. Figure 37 shows the ROC curve for this combined feature. 
Table 13: PERFORMANCE OF SYSTOLE ASC MEAN MULTIPLIED WITH SYSTOLE ASF

RMS

\begin{tabular}{|l|l|l|l|l|l|}
\hline Feature & Location & $\boldsymbol{J}_{\boldsymbol{O}}$ & Accuracy & Specificity & Sensitivity \\
\hline \multirow{4}{ASC_{S}}{$* A S F_{R M S, S}$} & 1 & 33.45 & 76 & 96 & 56 \\
\cline { 2 - 6 } & 2 & 39.24 & 78 & 100 & 56 \\
\cline { 2 - 6 } & 3 & 48.27 & 80 & 100 & 60 \\
\cline { 2 - 6 } & 4 & 14.66 & 90 & 92 & 92 \\
\hline
\end{tabular}

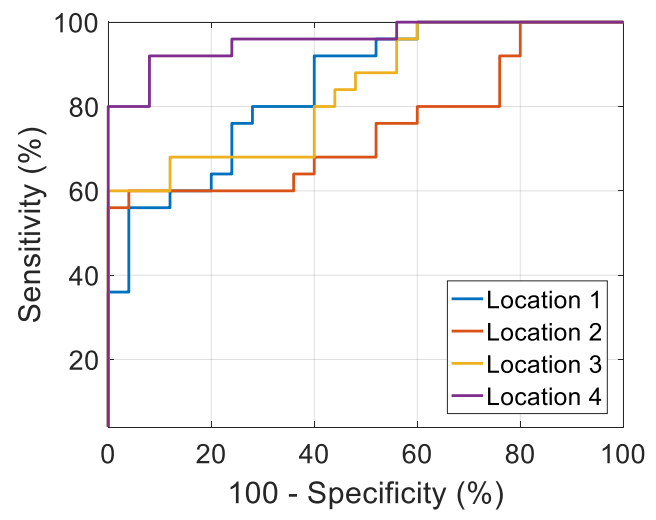

Figure 37: ROC curve of Systole ASC mean multiplied with systole ASF RMS. Greatest area-under-the curve is achieved at Location 4 distal to the stenosis. 


\section{SUMMARY OF STENOSIS DETECTION PERFORMANCE}

Our in vitro tests on vascular phantoms spanned a range of simulated stenosis severity (10-85\%) over blood flows ranging from $700-1200 \mathrm{~mL} / \mathrm{min}$. These values were chosen to represent adequate blood flow in vascular accesses to support dialysis treatment. Despite having sufficient blood flow, however, accesses with significant stenosis $(>50 \%)$ represented patients who would be at-risk for clotting or rapid stenosis progression due to cell growth. The clinical challenge, in this case, is that patients with significant stenosis still receiving dialysis treatment might not be identified. Phonoangiographic detection of stenosis location, and severity, could help to screen at-risk patients as they are receiving treatment. Therefore, this work had two primary goals: to detect the location of a stenotic lesion, and to detect stenosis $>50 \%$ using PAGs, regardless of the level of blood flow in the vascular access.

For stenosis detection, these results demonstrate two important outcomes. First, for all levels of stenosis and all blood flow rates, many ASC and ASF features were statistically different at recording sites $1 \mathrm{~cm}$ apart along the direction of blood flow. This suggests that multiple recordings are needed to accurately measure PAGs from a vascular access. Most previous work in this field has only taken a single recording at the site of a known lesion, or dual recordings at the arterial anastomosis and in the venous outflow tract [33], [35], [36], [42], [44], [46]. The need for multiple locations is highlighted by our second outcome, which showed that stenoses can be easily localized within $1 \mathrm{~cm}$ by analyzing changes in ASC or ASF features between successive recording sites. Because stenosis induces turbulent flow in the region 0.6-1.8 $\mathrm{cm}$ distal to the lesion (assuming a vessel diameter of $6 \mathrm{~mm}$ ), this outcome is supported by the current understanding of hemodynamics in vascular access [48], [49].

In terms of stenosis classification, we demonstrated a simple threshold-based binary classifier based on a combined feature. Because the $A S F_{R M S}$ feature had good sensitivity and $\overline{A S C}_{S}$ had good specificity, multiplying these features yielded a combined feature with improved sensitivity and 
specificity. Compared with more complex training-based classifiers, a simple threshold-based classifier is potentially more generalizable and less susceptible to over-fitting. 


\section{CONCLUSION}

Failure of vascular access is one of the main causes of hospital visits for a hemodialysis patient. In this thesis we have described a promising way to detect vascular accesses at-risk for sudden clotting or thrombosis. This method relies on the properties of turbulent blood flow, novel signal processing for feature extraction, and custom sensors for recording bruits simultaneously from multiple locations on a vascular access. Combining all these elements, we were able to localize stenosis within $1 \mathrm{~cm}$ of the true location and differentiate degree of stenosis into three grades (mild, moderate and severe).

PAG signal processing started with sub-band frequency domain linear prediction to enhance the systolic phase. Sub-band weights were multiplied with the FDLP signal to enhance the interpulse intervals and attenuate artifacts. After filtering, we computed the wavelet coefficients using the Morlet wavelet over 6 octaves with 12 voices/octave. Using the wavelet coefficients, we calculated features from two spectral waveforms-Auditory Spectral Flux (ASF) and Auditory Spectral Centroid (ASC). To improve the performance of these features, we segmented the PAG signal into systole and diastole phase.

Thin, flexible skin-contacting microphone arrays were developed using custom sensors based on PVDF transducers. Four sensor sizes $(2,4,8,16 \mathrm{~mm})$ were tested and $2 \mathrm{~mm}$ aperture size was chosen to be the best candidate for array integration due to the small size and wide acoustic bandwidth. We tested three backing materials (silicone gel, PDMS and air) and silicone gel had the best recording fidelity. A 5-channel flexible multi-channel sensor was constructed to record simultaneously from sites spaced $1 \mathrm{~cm}$ apart.

To validate stenosis detection performance, the array of transducers was tested on vascular stenosis phantoms with degree of stenosis (DOS) $10-80 \%$ and flow 700-1,200 mL/min. Recording sites were positioned to record bruits proximally and distally to the phantom stenosis lesion (4 locations: 1 proximal, 1 at stenosis and 2 distal). Independent to flow rate and DOS, each sensor 
site recorded unique spectral features $(p<0.033$ and $p<0.001)$. Large changes in mean systolic ASC between adjacent sites (e.g. drop from 323 to $223 \mathrm{~Hz}$ ), allowed detection of stenosis location in all phantoms within $1 \mathrm{~cm}$. The effect of DOS was to reduce the systole-diastole difference in mean ASC value, producing a nearly $4 \mathrm{x}$ decrease in systole-diastole ASC difference. This effect was leveraged by a simple binary classification method, which used a feature threshold to classify DOS above or below $50 \%$ for PAGs recorded at the site proximal to detected stenosis. The optimized performance of the classifier was excellent, achieving an accuracy of $90 \%$, specificity of $92 \%$ and sensitivity of $92 \%$.

Because PAGs can be collected non-invasively directly before dialysis treatment, they offer a potential method for screening of vascular accesses at risk for sudden clotting. However, analysis of PAGs includes several challenges, including sensor array design and signal processing of complex auditory signals with wide dynamic range. We have summarized the former work in this field, and highlighted our recent accomplishments validating new sensor topologies and signal processing approaches on the bench. These preliminary results suggest that simple threshold-based classification can achieve good detection of stenosis. Future work could include correlated comparisons between multiple sensor sites and the use of more complex machine-learning classifiers to further improve the stenosis detection capabilities of phonoangiographic analysis. 


\section{APPENDIX A. CLASSIFICATION OF LOCATION USING SIMPLE THESHOLDING}

The difference in $\overline{A S C}_{S}$ between adjacent locations was calculated to estimate the most likely location of the stenosis. The interval plot (Figure 38) indicates that there is a positive shift between the $\overline{A S C}_{S}$ differences from proximal to distal locations. In this experiment, the actual stenosis is located directly at location 2, location 1 is taken $1 \mathrm{~cm}$ proximal, and locations 3 and are 1 and $2 \mathrm{~cm}$ distal to stenosis. In general, differences between locations 3 and 4 are positive by at least $70 \mathrm{~Hz}$ while the other site differences are negative. This suggests that a simple threshold difference of 70 $\mathrm{Hz}$ in $\overline{A S C}_{S}$ between adjacent locations can identify stenosis proximally to the recording sites within $1-2 \mathrm{~cm}$ distance.

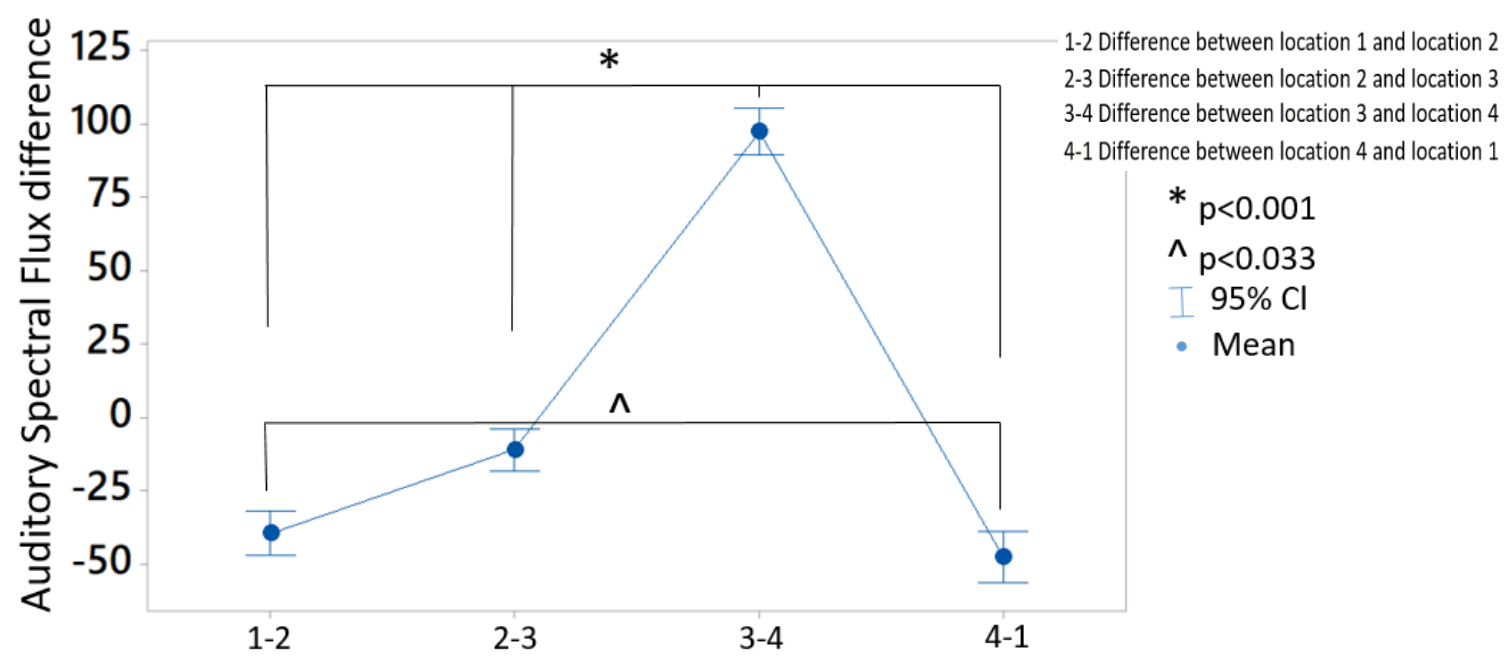

Figure 38: Difference in systole ASC mean from adjacent locations. 


\section{BIBLIOGRAPHY}

[1] H. I. Feldman, S. Kobrin, and A. Wasserstein, "Hemodialysis vascular access morbidity.," J. Am. Soc. Nephrol., vol. 7, no. 4, 1996.

[2] P. H. Sung, C. D. Kan, W. L. Chen, L. S. Jang, and J. F. Wang, "Hemodialysis vascular access stenosis detection using auditory spectro-temporal features of phonoangiography," Med. Biol. Eng. Comput., vol. 53, no. 5, pp. 393-403, 2015.

[3] R. L. Pisoni, L. Zepel, F. K. Port, and B. M. Robinson, "Trends in US Vascular Access Use, Patient Preferences, and Related Practices: An Update From the US DOPPS Practice Monitor With International Comparisons," Am. J. Kidney Dis., vol. 65, no. 6, pp. 905-915, Jun. 2015.

[4] "Hemodialysis Adequacy Peritoneal Dialysis Adequacy Vascular Access A Curriculum for CKD Risk Reduction and Care Kidney Learning System (KLS) ${ }^{\mathrm{TM}} 2006$ Updates Clinical Practice Guidelines and Recommendations."

[5] B. S. Dixon et al., "Effect of dipyridamole plus aspirin on hemodialysis graft patency.," $N$. Engl. J. Med., vol. 360, no. 21, pp. 2191-201, May 2009.

[6] M. Allon and I. D. Maya, "Hemodialysis arteriovenous graft dysfunction and failure UpToDate," Jan 29, $2018 . \quad$ [Online]. Available: https://www.uptodate.com/contents/hemodialysis-arteriovenous-graft-dysfunction-andfailure\#H349842688. [Accessed: 15-Jan-2019].

[7] C. D. Miller, M. L. Robbin, J. Barker, and M. Allon, "Comparison of arteriovenous grafts in the thigh and upper extremities in hemodialysis patients.," J. Am. Soc. Nephrol., vol. 14, no. 11, pp. 2942-7, Nov. 2003.

[8] J. C. Harms, S. Rangarajan, C. J. Young, J. Barker-Finkel, and M. Allon, "Outcomes of 
arteriovenous fistulas and grafts with or without intervention before successful use.," $J$. Vasc. Surg., vol. 64, no. 1, pp. 155-62, Jul. 2016.

[9] Vascular Access Work Group, "Clinical practice guidelines for vascular access.," Am. J. Kidney Dis., vol. 48 Suppl 1, pp. S248-73, Jul. 2006.

[10] M. L. Robbin, R. F. Oser, J. Y. Lee, G. R. Heudebert, S. T. Mennemeyer, and M. Allon, "Randomized comparison of ultrasound surveillance and clinical monitoring on arteriovenous graft outcomes.," Kidney Int., vol. 69, no. 4, pp. 730-5, Feb. 2006.

[11] M. Allon and M. L. Robbin, "Hemodialysis vascular access monitoring: current concepts.," Hemodial. Int., vol. 13, no. 2, pp. 153-62, Apr. 2009.

[12] A. Besarab et al., "Simplified measurement of intra-access pressure.," J. Am. Soc. Nephrol., vol. 9, no. 2, pp. 284-9, Feb. 1998.

[13] N. M. Krivitski, "Theory and validation of access flow measurement by dilution technique during hemodialysis.," Kidney Int., vol. 48, no. 1, pp. 244-50, Jul. 1995.

[14] N. R. Neyra et al., "Change in access blood flow over time predicts vascular access thrombosis," Kidney Int., vol. 54, no. 5, pp. 1714-1719, Nov. 1998.

[15] R. E. May et al., "Predictive measures of vascular access thrombosis: A prospective study," Kidney Int., vol. 52, no. 6, pp. 1656-1662, Dec. 1997.

[16] J. Sands, D. Glidden, and C. Miranda, "Hemodialysis access flow measurement. Comparison of ultrasound dilution and duplex ultrasonography.," ASAIO J., vol. 42, no. 5, pp. M899-901.

[17] J. H. Seo, “A coupled flow-acoustic computational study of bruits from a modeled stenosed artery." 
[18] G. W. Duncan, J. O. Gruber, C. F. Dewey, G. S. Myers, and R. S. Lees, "Evaluation of Carotid Stenosis by Phonoangiography," N. Engl. J. Med., vol. 293, no. 22, pp. 1124-1128, Nov. 1975.

[19] R. M. Rosen, S. P. Parthasarathy, A. F. Turner, D. H. Blankenhorn, and E. J. Roschke, “Phonoangiography by autocorrelation.," Circulation, vol. 55, no. 4, pp. 626-33, Apr. 1977.

[20] C.-D. Kan, W.-L. Chen, J.-F. Wang, P.-H. Sung, and L.-S. Jang, "Phonographic signal with a fractional-order chaotic system: A novel and simple algorithm for analyzing residual arteriovenous access stenosis View project Stenosis Detection using Burg Method with Autoregressive Model for Hemodialysis Patients View project Po-Hsun Sung Harman Hemodialysis vascular access stenosis detection using auditory spectro-temporal features of phonoangiography," 2015.

[21] S. J. A. Majerus et al., "Bruit-enhancing phonoangiogram filter using sub-band autoregressive linear predictive coding," pp. 4-7, 2000.

[22] T. Shinzato, S. Nakai, I. Takai, T. Kato, I. Inoue, and K. Maeda, "A new wearable system for continuous monitoring of arteriovenous fistulae.," ASAIO J., vol. 39, no. 2, pp. 137-40.

[23] A. E. Kalange and S. A. Gangal, "Piezoelectric Sensor for Human Pulse Detection," Def. Sci. J., vol. 57, no. 1, pp. 109-114, Jan. 2007.

[24] C. N. Teague et al., "Novel Methods for Sensing Acoustical Emissions From the Knee for Wearable Joint Health Assessment," IEEE Trans. Biomed. Eng., vol. 63, no. 8, pp. 15811590, Aug. 2016.

[25] R. S. Hallikar, S. Munshi, M. Uttara, K. Padmaraju, R. V. C. Engineering, and J. N. T. U. Kakinada, "Design and Implementation of Multichannel Piezoelectric Acoustic Sensor," no. 2014 , p. $2015,2015$. 
[26] K. F. Lei, Y. Z. Hsieh, Y. Y. Chiu, and M. H. Wu, "The structure design of piezoelectric poly(vinylidene fluoride) (PVDF) polymer-based sensor patch for the respiration monitoring under dynamic walking conditions," Sensors (Switzerland), vol. 15, no. 8, pp. 18801-18812, 2015.

[27] Y.-Y. Chiu, W.-Y. Lin, H.-Y. Wang, S.-B. Huang, and M.-H. Wu, "Development of a piezoelectric polyvinylidene fluoride (PVDF) polymer-based sensor patch for simultaneous heartbeat and respiration monitoring," Sensors Actuators A Phys., vol. 189, pp. 328-334, Jan. 2013.

[28] Y. Jiang et al., "A PVDF-based flexible cardiorespiratory sensor with independently optimized sensitivity to heartbeat and respiration," Procedia Eng., vol. 5, pp. 1466-1469, Jan. 2010.

[29] M. Allon and M. L. Robbin, "Hemodialysis vascular access monitoring: current concepts.," Hemodial. Int., vol. 13, no. 2, pp. 153-62, Apr. 2009.

[30] Y.-C. Du, W.-L. Chen, C.-H. Lin, C.-D. Kan, and M.-J. Wu, "Residual Stenosis Estimation of Arteriovenous Grafts Using a Dual-Channel Phonoangiography With Fractional-Order Features," IEEE J. Biomed. Heal. Informatics, vol. 19, no. 2, pp. 590-600, Mar. 2015.

[31] Y.-C. Du, C.-D. Kan, W.-L. Chen, and C.-H. Lin, "Estimating Residual Stenosis for an Arteriovenous Shunt Using a Flexible Fuzzy Classifier," Comput. Sci. Eng., vol. 16, no. 6, pp. 80-91, Nov. 2014.

[32] M.-J. Wu et al., "Dysfunction Screening in Experimental Arteriovenous Grafts for Hemodialysis Using Fractional-Order Extractor and Color Relation Analysis," Cardiovasc. Eng. Technol., vol. 6, no. 4, pp. 463-473, Dec. 2015.

[33] H. A. Mansy, S. J. Hoxie, N. H. Patel, and R. H. Sandler, "Computerised analysis of 
auscultatory sounds associated with vascular patency of haemodialysis access.," Med. Biol. Eng. Comput., vol. 43, no. 1, pp. 56-62, Jan. 2005.

[34] H.-Y. Hsien-Yi Wang, C.-H. Cho-Han Wu, C.-Y. Chien-Yue Chen, and B.-S. Bor-Shyh Lin, "Novel Noninvasive Approach for Detecting Arteriovenous Fistula Stenosis," IEEE Trans. Biomed. Eng., vol. 61, no. 6, pp. 1851-1857, Jun. 2014.

[35] W.-L. Chen, C.-H. Lin, T. Chen, P.-J. Chen, and Chung-Dann Kan, "Stenosis Detection using Burg Method with Autoregressive Model for Hemodialysis Patients," J. Med. Biol. Eng., vol. 33, no. 4, p. 356, 2013.

[36] Y. M. Akay, M. Akay, W. Welkowitz, J. L. Semmlow, and J. B. Kostis, "Noninvasive acoustical detection of coronary artery disease: a comparative study of signal processing methods," IEEE Trans. Biomed. Eng., vol. 40, no. 6, pp. 571-578, Jun. 1993.

[37] P. V. Obando and B. Mandersson, "Frequency tracking of resonant-like sounds from audio recordings of arterio-venous fistula stenosis," in 2012 IEEE International Conference on Bioinformatics and Biomedicine Workshops, 2012, pp. 771-773.

[38] P. O. Vesquez, M. M. Marco, and B. Mandersson, “Arteriovenous fistula stenosis detection using wavelets and support vector machines," in 2009 Annual International Conference of the IEEE Engineering in Medicine and Biology Society, 2009, pp. 1298-1301.

[39] Y.-N. Wang, C.-Y. Chan, and S.-J. Chou, "The Detection of Arteriovenous Fistula Stenosis for Hemodialysis Based on Wavelet Transform," 2011.

[40] T. Sato, K. Tsuji, N. Kawashima, T. Agishi, and H. Toma, "Evaluation of blood access dysfunction based on a wavelet transform analysis of shunt murmurs," J. Artif. Organs, vol. 9, no. 2, pp. 97-104, Jun. 2006.

[41] W.-L. Chen, C.-D. Kan, C.-H. Lin, W.-L. Chen, C.-D. Kan, and C.-H. Lin, "Arteriovenous 
Shunt Stenosis Evaluation Using a Fractional-Order Fuzzy Petri Net Based Screening System for Long-Term Hemodialysis Patients,” J. Biomed. Sci. Eng., vol. 07, no. 05, pp. 258-275, Apr. 2014.

[42] M. Gram et al., "Stenosis Detection Algorithm for Screening of Arteriovenous Fistulae," 2011, pp. 241-244.

[43] A. Todo et al., "Frequency analysis of shunt sounds in the arteriovenous fistula on hemodialysis patients," in The 6th International Conference on Soft Computing and Intelligent Systems, and The 13th International Symposium on Advanced Intelligence Systems, 2012, pp. 1113-1118.

[44] W.-L. Chen, T. Chen, C.-H. Lin, P.-J. Chen, and C.-D. Kan, "Phonographic signal with a fractional-order chaotic system: a novel and simple algorithm for analyzing residual arteriovenous access stenosis," Med. Biol. Eng. Comput., vol. 51, no. 9, pp. 1011-1019, Sep. 2013.

[45] M. M. Munguia and B. Mandersson, "Analysis of the vascular sounds of the arteriovenous fistula's anastomosis," in 2011 Annual International Conference of the IEEE Engineering in Medicine and Biology Society, 2011, pp. 3784-3787.

[46] M. Grochowina, L. Leniowska, and P. Dulkiewicz, "Application of Artificial Neural Networks for the Diagnosis of the Condition of the Arterio-venous Fistula on the Basis of Acoustic Signals," pp. 400-411, 2014.

[47] L. Rousselot, "Acoustical Monitoring of Model System for Vascular Access in Haemodialysis.” 2014.

[48] S. Gaupp, Y. Wang, T. V How, and P. J. Fish, "Characterisation of vortex shedding in vascular anstomosis models using pulsed doppler ultrasound," 1999. 
[49] R. Gårdhagen, Turbulent Flow in Constricted Blood Vessels Quantification of Wall Shear Stress Using Large Eddy Simulation. .

[50] B. Panda, S. Chin, S. Mandal, and S. Majerus, "Skin-Coupled PVDF Microphones for Noninvasive Vascular Blood Sound Monitoring," in 2018 IEEE Signal Processing in Medicine and Biology Symposium (SPMB), 2018, pp. 1-4.

[51] A. Devices, “Wide Supply Dual, $17 \mathrm{MHz}$, Rail-to-Rail FET Input Amplifier.”

[52] “TLV900x Low-Power, RRIO, 1-MHz Operational Amplifier for Cost-Sensitive Systems," 2017.

[53] by Hank Zumbahlen, "Mini Tutorial Multiple Feedback Filters.”

[54] S. J. A. Majerus, T. Knauss, S. Mandal, G. Vince, and M. S. Damaser, "Bruit-enhancing phonoangiogram filter using sub-band autoregressive linear predictive coding," in 2018 40th Annual International Conference of the IEEE Engineering in Medicine and Biology Society (EMBC), 2018, pp. 1416-1419.

[55] M. Athineos and D. P. W. E. Labrosa, "FREQUENCY-DOMAIN LINEAR PREDICTION FOR TEMPORAL FEATURES.”

[56] M. Athineos and D. P. W. Ellis, “Autoregressive Modeling of Temporal Envelopes," IEEE Trans. Signal Process., vol. 55, no. 11, pp. 5237-5245, Nov. 2007.

[57] G. Tzanetakis and P. Cook, "Musical Genre Classification of Audio Signals," IEEE Trans. SPEECH AUDIO Process., vol. 10, no. 5, p. 293, 2002.

[58] S. Chin, B. Panda, M. S. Damaser, and S. J. A. Majerus, "Stenosis Characterization and Identification for Dialysis Vascular Access," pp. 0-4.

[59] K. L. Gould, "Effects of stenosis on coronary flow," Cleve. Clin. J. Med., vol. 47, no. 3, pp. 
140-144, 2013.

[60] D. Bluestein, C. Gutierrez, M. Londono, and R. T. Schoephoerster, "Vortex Shedding in Steady Flow Through a Model of an Arterial Stenosis and Its Relevance to Mural Platelet Deposition,” Ann. Biomed. Eng., vol. 27, no. 6, pp. 763-773, Nov. 1999.

[61] T. Fawcett, “An introduction to ROC analysis,” Pattern Recognit. Lett., vol. 27, no. 8, pp. 861-874, Jun. 2006.

[62] M. D. Ruopp, N. J. Perkins, B. W. Whitcomb, and E. F. Schisterman, "Youden Index and optimal cut-point estimated from observations affected by a lower limit of detection.," Biom. J., vol. 50, no. 3, pp. 419-30, Jun. 2008.

[63] E. F. Schisterman, N. J. Perkins, A. Liu, and H. Bondell, "Optimal Cut-point and Its Corresponding Youden Index to Discriminate Individuals Using Pooled Blood Samples,” Epidemiol. • vol. 16, no. 1, 2005.

[64] R. Parikh, A. Mathai, S. Parikh, G. Chandra Sekhar, and R. Thomas, "Understanding and using sensitivity, specificity and predictive values.," Indian J. Ophthalmol., vol. 56, no. 1, pp. 45-50, 2008. 